\title{
STELLAR ARCHAEOLOGY: A KECK PILOT PROGRAM ON EXTREMELY METAL-POOR STARS FROM THE HAMBURG/ESO SURVEY. III. THE LEAD (Pb) STAR HE 0024-2523 1,2
}

\author{
Sara Lucatello, ${ }^{3,4}$ Raffaele Gratton, ${ }^{3}$ Judith G. Cohen, ${ }^{5}$ Timothy C. Beers, ${ }^{6}$ Norbert Christlieb, ${ }^{7,8,9}$ \\ Eugenio Carretta, ${ }^{3}$ And Solange Ramírez ${ }^{5}$ \\ Received 2002 September 16; accepted 2002 October 31
}

\begin{abstract}
We present a detailed abundance analysis, including spectral syntheses, of a very metal-poor $([\mathrm{Fe} / \mathrm{H}]$ $\simeq-2.7$ ) peculiar main-sequence star (HE 0024-2523) detected during the course of the Keck Pilot Program. Radial velocities of this star were obtained during four different observing runs over a time span of $1.1 \mathrm{yr}$ and demonstrate that it is clearly a short-period spectroscopic binary. An orbital solution was obtained, and orbital parameters were determined with high precision. The rotational velocity was also measured $\left(v_{\text {rot }} \sin i=9.7 \pm 1.5 \mathrm{~km} \mathrm{~s}^{-1}\right)$; rotation appears likely to be synchronous with the orbit. The abundance analysis and spectral syntheses indicate that the object is a $\mathrm{CH}$ star characterized by extreme $s$-process enrichment, likely due to mass accretion from an evolved companion that has now probably become a white dwarf. The lead $(\mathrm{Pb})$ abundance of (HE 0024-2523) is very high, the same as that of the recently discovered lead-rich, metal-poor star CS 29526-110, $[\mathrm{Pb} / \mathrm{Fe}]=+3.3$. The abundance ratio of the heavy to light $s$-elements, as characterized by $\mathrm{Pb}$ and $\mathrm{Ba},[\mathrm{Pb} / \mathrm{Ba}]=+1.9$, is the highest yet found for any metal-poor star and is about 0.7 dex higher than that of CS 29526-110. On the basis of the measured isotopic ratio of carbon $\left(C^{12} / C^{13} \sim 6\right)$ we argue that the mass donor must have had an original mass of at least $\sim 3 M_{\odot}$. The unusually short period of this $\mathrm{CH}$ star suggests that it underwent a past common-envelope phase with its evolved companion. Our results are compared with the latest available models for asymptotic giant branch yields and $s$-process nucleosynthesis. We also discuss the possible connection between HE 0024-2523, the lithium depletion of halo stars, and halo blue straggler formation.
\end{abstract}

Key words: binaries: spectroscopic — stars: AGB and post-AGB — stars: carbon — stars: chemically peculiar

On-line material: color figures

\section{INTRODUCTION}

One of the surprising results of the hitherto largest widefield spectroscopic survey for metal-poor stars, the HK survey (Beers, Preston, \& Shectman 1992; Beers 1999), is the high frequency of carbon-enhanced stars among very metalpoor stars. Norris, Ryan, \& Beers (1997a) found that $14 \%$ of the HK survey $\operatorname{stars}^{10}$ with $[\mathrm{Fe} / \mathrm{H}]<-2.5$ have stronger than normal $\mathrm{G}$ bands. More recent results, based on a larger sample, suggest that the frequency of carbon-enhanced stars (i.e., stars with $[\mathrm{C} / \mathrm{Fe}]>+1.0$ ) among metal-poor stars

\footnotetext{
${ }^{1}$ Based in part on observations obtained at the W. M. Keck Observatory, which is operated jointly by the California Institute of Technology, the University of California, and NASA.

${ }^{2}$ Based in part on observations collected at the European Southern Observatory, Paranal, Chile (ESO program 167.D-0173[A]).

${ }^{3}$ Osservatorio Astronomico di Padova, Instituto Nazionale di Astrofisica, vicolo dell'Osservatorio 5, I-35122 Padova, Italy.

${ }^{4}$ Dipartimento di Astronomia, Università di Padova, vicolo dell'Osservatorio 2, I-35122 Padova, Italy.

${ }^{5}$ Palomar Observatory, Mail Stop 105-24, California Institute of Technology, 1201 East California Boulevard, Pasadena, CA 91125.

${ }^{6}$ Department of Physics and Astronomy, Michigan State University, East Lansing, MI 48824-1116.

${ }^{7}$ Hamburger Sternwarte, Gojenbergsweg 112, D-21029 Hamburg, Germany.

${ }^{8}$ Department of Astronomy and Space Physics, Uppsala University, Box 524, SE-75239 Uppsala, Sweden.

${ }_{9}^{9}$ Marie Curie Fellow, on sabbatical leave from Hamburger Sternwarte.

${ }^{10}$ We use the usual spectroscopic notation: $\log n(\mathrm{~A})$ is the abundance (by number) of the element $\mathrm{A}$ on the usual scale where $\log n(\mathrm{H})=12$, while $[\mathrm{A} / \mathrm{H}]$ denotes the logarithmic ratio of the abundances of elements $\mathrm{A}$ and $\mathrm{H}$ in the star, minus the same quantity in the Sun.
}

with $[\mathrm{Fe} / \mathrm{H}]<-2.5$ is perhaps as high as $25 \%$. These findings are confirmed by the Hamburg/ESO objective-prism survey (Wisotzki et al. 2000; Christlieb et al. 2001a, 2001b), where $\sim 30 \%$ of the metal-poor candidates appear to be carbon-enhanced stars.

Despite extensive investigations of this class of objects by means of high-resolution spectroscopy (Norris et al. 1997a, Norris, Ryan, \& Beers 1997b, 2002; Bonifacio et al. 1998; Hill et al. 2000; Aoki et al. 2002b), the origin of carbon in these stars still remains unclear. The extremely metal-poor star CS 22892-52 (McWilliam et al. 1995; $[\mathrm{Fe} / \mathrm{H}]=-3.1$ ) exhibits an overabundance of carbon $([\mathrm{C} / \mathrm{Fe}]=+1.0)$, as well as of $r$-process elements (Sneden et al. 1994, 1996, 2000; $[r / \mathrm{Fe}] \sim+1.6)$, hinting at a common mechanism for the enrichment, in particular, by supernovae of Type II, as discussed by Aoki et al. (2002b). However, Aoki et al. also found a class of carbon-rich, metal-poor stars without neutron capture element excess, arguing against a common enrichment mechanism. Furthermore, a second, recently discovered very metal-poor star with strong $r$-process enrichment, CS 31082-001 (Cayrel et al. 2001), is only very slightly carbon enhanced, $[\mathrm{C} / \mathrm{Fe}]=+0.2$ (Hill et al. 2002).

A different scenario for the origin of carbon in these stars is that proposed for the moderately metal-poor classical $\mathrm{CH}$ stars $([\mathrm{Fe} / \mathrm{H}] \sim-1.5)$, in which the carbon-enhanced star is a member of a wide binary system that accreted material from a former primary, during the asymptotic giant branch (AGB) phase of the latter, as described by McClure \& Woodsworth (1990). However, while all CH stars are indeed members of binary systems (McClure \& Woodsworth 1990) and radial velocity variations have been reported (and 
periods determined) for some carbon-enhanced, metal-poor stars (CEMP stars; Norris et al. 1997a; Aoki et al. 2000; Preston \& Sneden 2001), the latter authors found that three of their CEMP subgiants do not show any radial velocity variations greater than $0.4 \mathrm{~km} \mathrm{~s}^{-1}$ over a period of 8 years, strongly arguing against the mass transfer scenario for these three stars. Furthermore, in this scenario it is expected that the star we observe today should display an enrichment of $s$-process elements, produced by the former primary in its AGB phase, while the CEMP star CS 22957-027 (Norris et al. 1997b; Bonifacio et al. 1998), as well as the previously mentioned class of stars found by Aoki et al., does not exhibit this behavior.

The CEMP stars that do show s-process enrichment provide the unique opportunity to study an extinct generation of extremely metal-poor AGB stars that left their fingerprints on their less massive companions. These stars provide strong observational constraints for theoretical models of the structure, evolution, and nucleosynthesis of early-epoch AGB stars and permit studies of the $s$-process operating at very low metallicities. Recent calculations by Goriely \& Siess (2001) predict that efficient production of $s$-process elements takes place even at zero metallicity, despite the absence of iron seeds, provided that protons are mixed into carbon-rich layers. Proton mixing results in the formation of ${ }^{13} \mathrm{C}$, which is a strong neutron source because of the reaction ${ }^{13} \mathrm{C}(\alpha, n){ }^{16} \mathrm{O}$. The recent discovery of CEMP stars with strong overabundances of $\mathrm{Pb}$ by Aoki et al. (2000) and Van Eck et al. (2001) is in concert with these predictions.

In two previous papers, Cohen et al. (2002, hereafter Paper I) and Carretta et al. (2002, hereafter Paper II), we analyzed a sample of extremely metal-poor stars from the Hamburg/ESO prism survey. As briefly discussed in Paper II, one of the objects, HE 0024-2523, exhibited a chemical signature typical of $\mathrm{CH}$ stars. The $G$ band of $\mathrm{CH}$ was found to be extremely strong, while the evidence of a large overabundance of neutron capture elements, coupled with an apparent lack of strong Eu lines, suggested that the object was enriched by the $s$-process. Furthermore, the previously reported detection of the $\mathrm{Pb}$ line at $4057.8 \AA$ suggested an enormous lead overabundance. To measure the abundances of carbon, the s-process elements, and the light element $\mathrm{N}$ with higher precision and to study the dynamical behavior of this peculiar object, more observations were needed. In this paper we present results from an analysis of several spectra obtained with the Keck I, VLT-UT2, and the Palomar Hale telescopes.

After summarizing our observations in $\S 2$, we describe the radial velocity measurements, the modeling of the radial velocity curve, and the computation of the orbital parameters in $\S 3$. In $\S 4$ we present the abundance analysis from equivalent widths and from spectral syntheses. We discuss our interpretation of these results in $\S 5$.

\section{OBSERVATIONS AND DATA REDUCTIONS}

A total of 18 spectra of HE 0024-2523 have been collected during several runs using three different instrumenttelescope combinations: UVES at VLT-UT2 Kueyen (Dekker et al. 2000), HIRES at Keck I (Vogt et al. 1994), and the double spectrograph at the Hale $5 \mathrm{~m}$ telescope at Palomar Mountain (Oke \& Gunn 1982).

The UVES observations were carried out during two different runs, on 2001 August 27, and in 2001 between Octo- ber 23 and 27. Three metal-poor field stars, G4-37, G12662 , and G141-19, were observed with the same setup, so that they could be used as templates to measure rotational velocities. The signal-to-noise ratio for the template spectra is $\sim 200 / 1$ per resolution element. The spectra have a resolution of $R=\lambda / \Delta \lambda \simeq 50,000$, where $\Delta \lambda$ refers to the width of the resolution elements. A dichroic beam splitter was employed, which distributed the light gathered with the telescope to the blue and red arms of UVES, covering a range from 3600 to $4800 \AA$ and from 5700 to $9500 \AA$, respectively. The slit width was fixed at $1^{\prime \prime}$ and the binning used $1 \times 2$, where 1 refers to the dispersion direction and 2 to the spatial one. The spectra were extracted and wavelength calibrated using the standard UVES pipeline. The bias and scattered light were subtracted from the spectra, flat-fielding was applied, and the extracted spectra were wavelength calibrated with arc lamp spectra taken during the same observing run; then the orders were blaze corrected and merged.

The Keck I HIRES spectra are described in detail in Paper I. They have a wavelength coverage from 3850 to $5400 \AA$ and were observed with a slit width of 0 "'86 and $1 \times 2$ binning on the CCD chip. The first five spectra were all collected during a single night, 2000 September 24. Two more spectra were obtained, one on 2001 September 27, and the other on 2001 September 30. All the spectra have a resolution of $R \simeq 45,000$, and their extraction and wavelength calibration were performed using FIGARO (Shortridge 1993). The reduction was similar to that performed on the UVES spectra, but in this case the orders were not merged.

The Palomar spectra were obtained during four consecutive nights, from 2001 October 24 to 27 , with a $1{ }^{\prime \prime} 0$ slit and $0.6 \AA$ pixels, yielding a spectral resolution of $R \simeq 3500$ over the spectral region 6100 to $6700 \AA$, with $\mathrm{H} \alpha$ the major spectral feature present in that range. FIGARO was again used to extract and wavelength calibrate the spectra. Several radial velocity standards (HD 2796, HD 38230, HD 171232, and CS 22950-046) were also observed to test the reliability of our measurements. Further details on the observations are listed in Table 1.

\section{DYNAMICS \\ 3.1. Data Analysis \\ 3.1.1. Radial Velocities}

Radial velocities were measured by fitting Gaussians to several prominent lines in different orders and adding the appropriate barycentric correction. The values measured for the radial velocity standards showed a systematic offset in the Palomar spectra of $\sim 4.8 \mathrm{~km} \mathrm{~s}^{-1}$, which was added to the measured radial velocities as a correction for instrumental effects. The measured heliocentric radial velocities are given in Table 1.

\subsubsection{Rotational Velocities}

As mentioned in Paper I, the spectra of HE 0024-2523 were characterized by broader lines than those of the other program stars, suggesting a nonnegligible rotational velocity. In Paper I, we roughly estimated $v_{\text {rot }}$ to be $\sim 8 \mathrm{~km} \mathrm{~s}^{-1}$.

Here we improve upon this measurement. The rotational velocity was measured using a cross-correlation technique; the spectrum of the program star was cross-correlated against a few template spectra of nonrotating stars with similar atmospheric parameters. The cross-correlation peaks 
TABLE 1

Description OF THE ObSERVATIONS

\begin{tabular}{|c|c|c|c|c|c|c|}
\hline Date & Spec. $^{\mathrm{a}}$ & $\begin{array}{l}\text { Exp. time } \\
\text { (s) }\end{array}$ & $\mathrm{S} / \mathrm{N}^{\mathrm{b}}$ & $\mathrm{MJD}^{\mathrm{c}}$ & $\begin{array}{c}v_{r} \\
\left(\mathrm{~km} \mathrm{~s}^{-1}\right)\end{array}$ & $\begin{array}{c}\sigma \\
\left(\mathrm{km} \mathrm{s}^{-1}\right)\end{array}$ \\
\hline 2000 Sep 24 . & HIRES & 1200 & 40 & $51,811.386$ & -185.0 & 0.4 \\
\hline 2000 Sep 24 . & HIRES & 1200 & 40 & $51,811.397$ & -183.6 & 0.8 \\
\hline 2000 Sep $24 \ldots \ldots \ldots \ldots . . . .$. & HIRES & 1200 & 40 & $51,811.412$ & -186.6 & 0.7 \\
\hline 2000 Sep $24 \ldots \ldots \ldots \ldots \ldots$ & HIRES & 1200 & 40 & $51,811.427$ & -187.4 & 1.1 \\
\hline 2000 Sep 24 . & HIRES & 1200 & 40 & $51,811.442$ & -189.6 & 0.3 \\
\hline 2001 Sep 27. & HIRES & 1200 & 40 & $52,179.398$ & -135.8 & 1.1 \\
\hline 2001 Sep 30 . & HIRES & 1200 & 40 & $52,182.365$ & -125.6 & 1.2 \\
\hline 2001 Aug $27 \ldots \ldots \ldots \ldots . . .$. & UVES & 3600 & 80 & $52,148.352$ & -127.0 & 0.5 \\
\hline 2001 Oct $23 \ldots \ldots \ldots \ldots$ & UVES & 600 & 30 & $52,205.017$ & -218.2 & 0.7 \\
\hline 2001 Oct $23 \ldots \ldots \ldots \ldots$ & UVES & 1800 & 40 & $52,206.014$ & -136.2 & 0.4 \\
\hline 2001 Oct $25 \ldots \ldots \ldots \ldots . .$. & UVES & 600 & 20 & $52,207.010$ & -160.8 & 0.6 \\
\hline 2001 Oct $26 \ldots \ldots \ldots \ldots$ & UVES & 1800 & 35 & $52,208.161$ & -228.6 & 0.5 \\
\hline 2001 Oct $28 \ldots \ldots \ldots \ldots \ldots$ & UVES & 600 & 20 & $52,210.021$ & -133.9 & 0.8 \\
\hline 2001 Oct $28 \ldots \ldots \ldots \ldots . . .$. & UVES & 600 & 20 & $52,210.255$ & -148.2 & 0.9 \\
\hline 2001 Oct $25 \ldots \ldots \ldots \ldots \ldots$ & Palomar & 400 & 30 & $52,207.241$ & -186.2 & 5.0 \\
\hline 2001 Oct $26 \ldots \ldots \ldots \ldots . .$. & Palomar & 400 & 30 & $52,208.251$ & -227.8 & 5.0 \\
\hline 2001 Oct 27 .................. & Palomar & 400 & 30 & $52,209.218$ & -145.6 & 5.0 \\
\hline 2001 Oct $28 \ldots \ldots \ldots \ldots \ldots$ & Palomar & 400 & 30 & $52,210.216$ & -140.1 & 5.0 \\
\hline
\end{tabular}

a Spectrograph with which the observations were collected.

$\mathrm{b}$ The signal-to-noise ratio is per resolution element.

${ }^{c}$ Modified Julian Day, MJD = JD-2,400,000.5.

were fitted with a Gaussian curve; from the resulting FWHM the value of $v \sin i$ was calculated according to a relation calibrated on several other stars with well-known rotational velocities. A more detailed description of the procedure used can be found in Lucatello \& Gratton (2002). The spectrum used for this purpose was that with the highest signal-to-noise ratio, the UVES spectrum taken on 2001 August 27. Only the blue part was used to measure rotation, as it is not affected by telluric lines and exhibited the strongest signatures in the cross-correlations. The spectrum was first divided into single orders. Each order was then crosscorrelated against the corresponding orders of the three different templates using the IRAF task RV.FXCOR. From the resulting average FWHMs, after subtracting all the external sources of broadening (e.g., template contribution and instrumental broadening), we obtained $v \sin i=$ $9.7 \pm 1.5 \mathrm{~km} \mathrm{~s}^{-1}$. This value is strictly an upper limit, as it includes also nonrotational intrinsic stellar broadening, such as macroturbulence. However, as shown in Lucatello \& Gratton (2002), this procedure provides a good estimate of the value of the true $v \sin i$ when its value is larger than $\sim 6$ $\mathrm{km} \mathrm{s}^{-1}$.

\subsection{Modeling the Radial Velocity Curve}

To compute the orbital parameters for HE 0024-2523, which for the sake of clarity we will refer to hereafter as the primary star (the companion will be called the secondary), we employed the program ORBITSOLVER by J. S. Varga, G. A. Radford, D. W. Beggs, R. E. M. Griffin, and J. R. Lewis (R. E. M. Griffin 2002, private communication). This program adjusts all the elements of the orbit simultaneously in a least-squares solution (Griffin \& Cornell 1998).

The measurements of the radial velocities were fed into the program, along with the corresponding MJD of each of the observations. The values of the velocities were weighted according to their errors of observation. However, note that we did not use the individual errors, but rather an estimate of the average errors, $\sim 1 \mathrm{~km} \mathrm{~s}^{-1}$ for HIRES and UVES data and $\sim 5 \mathrm{~km} \mathrm{~s}^{-1}$ for the Palomar data. The initial values of the parameters were provided from direct examination of the data. The initial period was assumed to be four days, since we noted that the radial velocity was very similar for the two UVES observations taken at MJD $=52,206.014$ and 52,210.021. For the initial semiamplitude of the orbit we used half the difference between the highest and lowest velocity points, and for the epoch at zero phase we assumed the epoch of the first observation. A stable and reasonable solution was obtained after five iterations.

Initially, we assumed a circular orbit (i.e., $e=0$ ); after having refined the other orbital elements, we allowed the eccentricity to vary as well. Our best solutions for circular and noncircular orbits are provided in Table 2, along with their values before the last iteration. The short period suggests that the orbit should have an eccentricity close to zero.

Our computation of the orbital elements, solving for $e$ as well, obtained a very small value for $e$ and did not significantly change the other orbital parameters with respect to those obtained when adopting a circular orbit. Allowing the eccentricity to vary did not improve the solution significantly. In fact, the circular solution yielded smaller rms residuals, and hence we adopted a circular orbit. Figure 1 shows the radial velocity data together with the adopted solution, while Table 3 lists the observed values for the radial velocities along with their expected value from our orbital solution.

\subsection{Mass Assumptions and Consequences on the Orbital Parameters}

Although the values of the individual masses are not known, we can derive useful limits on them through considerations based on the available photometric and spectroscopic information for HE 0024-2523. 
TABLE 2

Our Best Orbital Solutions

\begin{tabular}{|c|c|c|c|}
\hline Orbital Element & Initial Value for Last Iteration & Computed Value & Standard Error \\
\hline \multicolumn{4}{|l|}{ Circular orbit: } \\
\hline 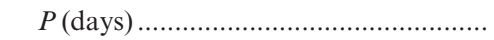 & 3.14 & 3.41263 & 0.00008 \\
\hline$T_{0}$ (days) & 51811.0 & 52059.596 & 0.005 \\
\hline 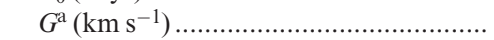 & 0.0 & -178.3 & 0.4 \\
\hline 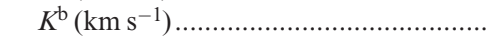 & 50.0 & 51.9 & 0.5 \\
\hline е & 0.0 & 0.0 & $\ldots$ \\
\hline Projected semiaxis, $a \sin i \ldots \ldots \ldots \ldots \ldots \ldots \ldots$ & $10^{6} \mathrm{~km}$ & 2.438 & 0.023 \\
\hline Mass function, $f(m)$ & $M_{\odot}$ & 0.0496 & 0.0014 \\
\hline \multicolumn{4}{|l|}{ Eccentric orbit: } \\
\hline 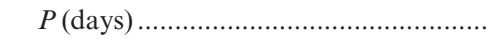 & 3.14 & 3.41265 & 0.00016 \\
\hline 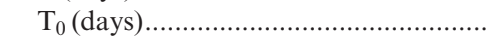 & 51811.0 & 52059.601 & 0.009 \\
\hline 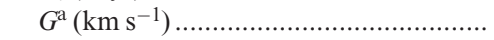 & 0.0 & -178.4 & 0.6 \\
\hline 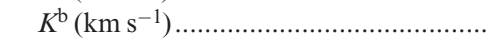 & 50.0 & 51.7 & 0.7 \\
\hline 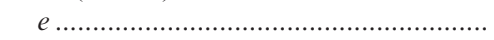 & 0.0 & 0.010 & 0.016 \\
\hline$\omega^{\mathrm{c}}$ & 0.0 & 329 & 95 \\
\hline Projected semiaxis, $a \sin i \ldots \ldots \ldots \ldots \ldots \ldots \ldots \ldots \ldots \ldots$ & $10^{6} \mathrm{~km}$ & 2.43 & 0.03 \\
\hline 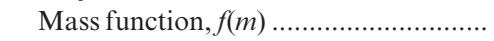 & $M_{\odot}$ & 0.0490 & 0.0020 \\
\hline Epoch of ascending node, $T$ (days) $\ldots \ldots .$. & & 52062.7 & 0.9 \\
\hline
\end{tabular}

aean radial velocity of the system, i.e., radial velocity of the barycenter.

${ }^{b}$ Semiamplitude of the radial velocity curve.

${ }^{c}$ Longitude of the ascending node.

\subsubsection{Primary Star Mass}

We begin by considering the mass of the primary. If we assume a constant spatial density for the extreme halo population, which is reasonable for distances up to a few kiloparsecs, and a uniform mass function (adopted since information on the mass of the observed star is lacking), ${ }^{11}$

${ }^{11}$ Note that these assumptions are not critical in the present discussion.

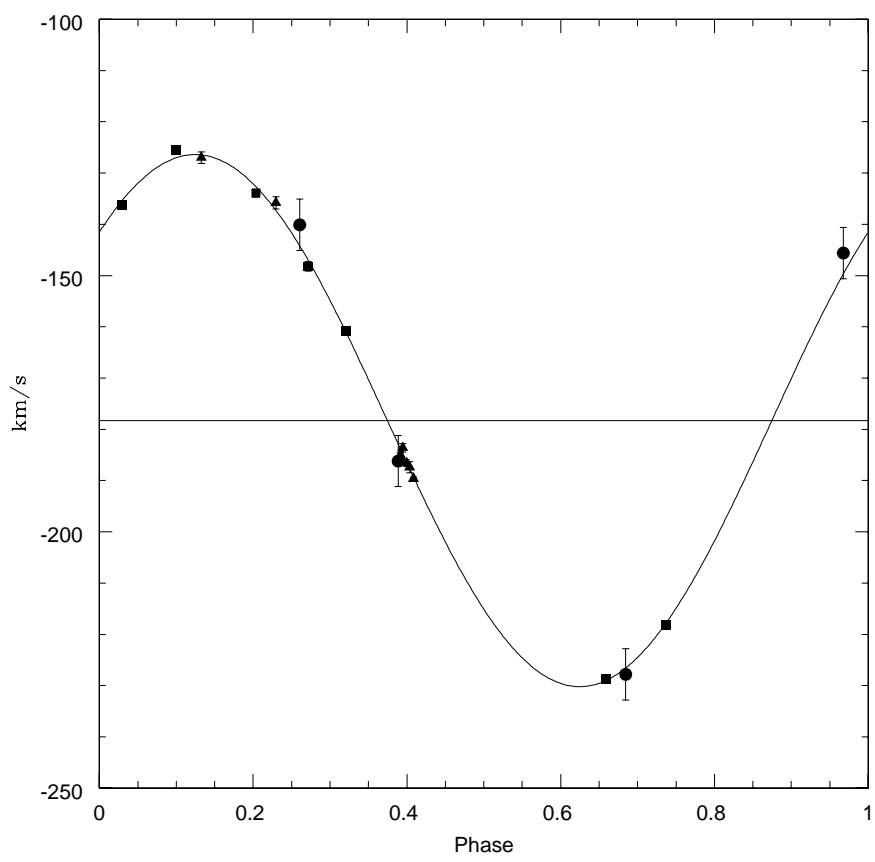

FIG. 1.-Radial velocity curve. The curve represents our best solution. The symbols are the data phased according to the period we derive (see Table 2 for details of the orbital parameters for our orbital solution): HIRES data (triangles), UVES data (squares), and Palomar data (circles). The radial velocity errors are shown for the Palomar spectra, where the lower spectral dispersion leads to much larger uncertainties. The HIRES and UVES spectra have errors comparable to the size of the symbols used. The horizontal line indicates the radial velocity of the barycenter. then the probability of observing a star of a given mass at the measured magnitude and color can be expressed by the formula

$$
P\left(M^{*}\right)=\frac{\sum_{i=1}^{2} \tau_{i}\left(M^{*}\right) \Delta V_{i}\left(M^{*}\right)}{\sum_{j=1}^{n} \sum_{i=1}^{2} \tau_{i}\left(M_{j}\right) \Delta V_{i}\left(M_{j}\right)},
$$

where $\tau_{i}\left(M_{j}\right)$ is the time spent in the $i$ th phase (with $i=1$ being the main-sequence phase and $i=2$ the subgiant phase) by the star of $j$ th mass within the restricted evolutionary phase allowed by the atmospheric parameters. $\Delta V(M)$ is the volume sampled by stars of the measured luminosity, which in turn depends on the intrinsic luminosity of the object. The integral in the denominator normalizes $P(M)$.

TABLE 3

Comparison of the Observed and Predicted Radial Velocities

\begin{tabular}{ccccc}
\hline \hline MJD & $\begin{array}{c}v_{o}{ }^{\mathrm{a}} \\
\left(\mathrm{km} \mathrm{s}^{-1}\right)\end{array}$ & Phase & $\begin{array}{c}v_{c}{ }^{\mathrm{b}} \\
\left(\mathrm{km} \mathrm{s}^{-1}\right)\end{array}$ & $\begin{array}{c}v_{o}-v_{c} \\
\left(\mathrm{~km} \mathrm{~s}^{-1}\right)\end{array}$ \\
\hline $51,811.39 \ldots \ldots \ldots \ldots$ & -185.0 & 0.267 & -183.9 & -1.1 \\
$51,811.40 \ldots \ldots \ldots \ldots$. & -183.6 & 0.270 & -184.9 & +1.3 \\
$51,811.41 \ldots \ldots \ldots \ldots$. & -186.6 & 0.275 & -186.4 & -0.2 \\
$51,811.43 \ldots \ldots \ldots \ldots$. & -187.4 & 0.279 & -187.8 & +0.4 \\
$51,811.44 \ldots \ldots \ldots \ldots$. & -189.6 & 0.284 & -189.2 & -0.4 \\
$52,148.35 \ldots \ldots \ldots \ldots$. & -127.0 & 0.008 & -126.5 & -0.5 \\
$52,179.40 \ldots \ldots \ldots \ldots$. & -135.8 & 0.105 & -137.4 & +1.6 \\
$52,182.37 \ldots \ldots \ldots \ldots$. & -125.6 & 0.975 & -127.1 & +1.5 \\
$52,205.02 \ldots \ldots \ldots \ldots$. & -218.2 & 0.612 & -217.8 & -0.4 \\
$52,206.01 \ldots \ldots \ldots \ldots$. & -136.2 & 0.905 & -135.5 & -0.7 \\
$52,207.01 \ldots \ldots \ldots \ldots$. & -160.8 & 0.196 & -161.2 & +0.4 \\
$52,208.16 \ldots \ldots \ldots \ldots$. & -228.6 & 0.534 & -229.1 & +0.5 \\
$52,210.02 \ldots \ldots \ldots \ldots$. & -133.9 & 0.079 & -132.6 & -1.3 \\
$52,210.26 \ldots \ldots \ldots \ldots$. & -148.2 & 0.147 & -147.1 & -1.1 \\
$52,207.24 \ldots \ldots \ldots \ldots$. & -186.2 & 0.264 & -182.9 & -3.3 \\
$52,208.25 \ldots \ldots \ldots \ldots$. & -227.8 & 0.560 & -226.6 & -1.2 \\
$52,209.22 \ldots \ldots \ldots \ldots$. & -145.6 & 0.843 & -149.6 & +4.0 \\
$52,210.22 \ldots \ldots \ldots \ldots$. & -140.1 & 0.136 & -144.2 & +4.1 \\
\hline
\end{tabular}

a Observed radial velocity.

${ }^{\mathrm{b}}$ Computed radial velocity from our adopted solution. 
To compute these quantities we used the $\mathrm{Y}^{2}$ tracks (Yi et al. 2001) for a mass interval $0.4<M / M_{\odot}<5$ with $0.1 M_{\odot}$ bins, with a metallicity $[\mathrm{Fe} / \mathrm{H}]=-3.3$, and $[\alpha / \mathrm{Fe}]=+0.3$. We assumed the $\pm 2 \sigma$ range around the measured temperature of HE $0024-2523$ (i.e., 6425 to $6825 \mathrm{~K}$ ) to specify the evolutionary phase and computed the time spent in this range. In this way we obtained two permitted intervals for each mass, one on the main sequence and the other along the subgiant branch. The number of stars of the corresponding mass observable at the measured magnitude was calculated from the theoretical tracks, assuming a bolometric magnitude for the Sun of $M_{b}=4.711$ and a bolometric correction for our star of $\mathrm{BC}(V)=-0.091$ (Lejeune, Cuisinier, \& Buser 1998).

The final probabilities were evaluated according to equation (1); Figure 2 provides a graphical representation of the results. In this figure a value of zero for the probability of a given mass indicates that the track for that mass does not cross the allowed region in $T_{\text {eff. }}$ This figure shows that the probability function drops abruptly at $0.9 M_{\odot}$ but seems to be roughly constant (at low but nonzero values) in the interval $0.95<M / M_{\odot}<2.85$. This behavior arises because as the time spent in the observed temperature region decreases, the intrinsic luminosity increases, hence the volume of space in which stars of such luminosity are observed at the measured magnitude increases.

Application of the ionization equilibrium for $\mathrm{Fe}$ supplies a further constraint, since it limits the acceptable

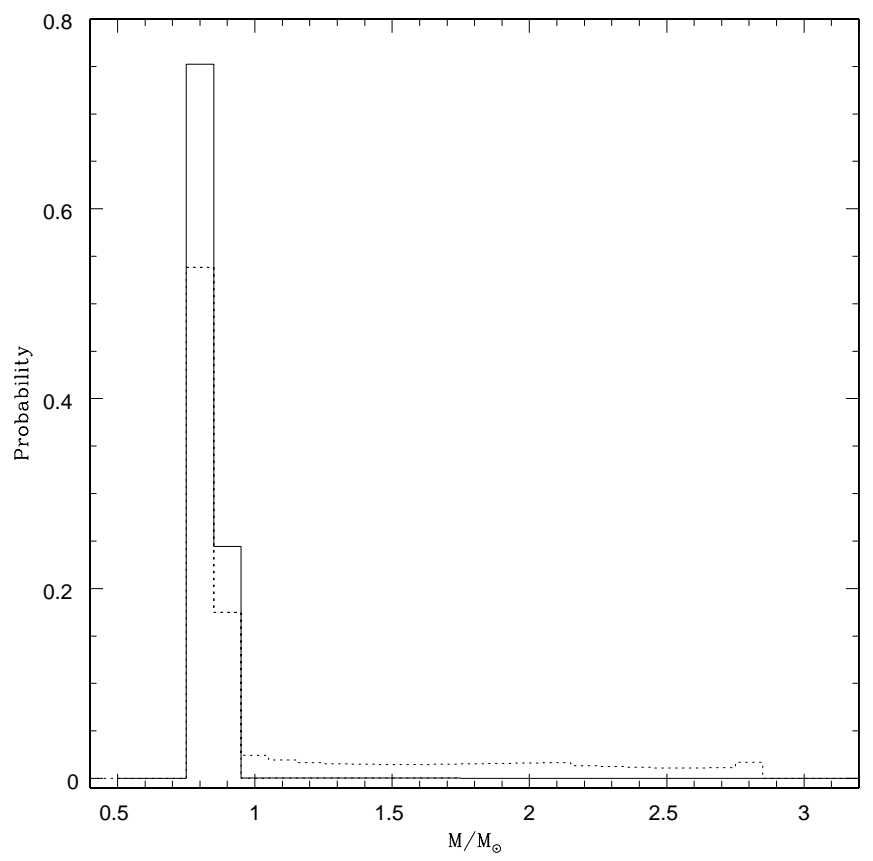

FIG. 2.-Solid-line histogram represents the normalized probability distribution for the mass of the observed star. It has been computed by using the $\mathrm{Y}^{2}$ tracks (Yi et al. 2001) and assuming constant stellar density (for a detailed description see text). The tracks for $M>2.8 M_{\odot}$ do not cross the allowed region of $T_{\text {eff }}$ and hence have a probability of zero. The probability function drops abruptly at $0.9 M_{\odot}$ but seems to be approximately flat in the interval $0.9<M / M_{\odot}<2.8$. This is due to the fact that as the time spent in the observed temperature region decreases, the intrinsic luminosity increases, which increases the volume of space in which stars of such luminosity are observed at the measured magnitude, and so the product of the two terms is roughly constant. The dashed-line histogram represents the probability distribution for the mass range allowed by the requirements on the gravity. values of surface gravity. The lower limit on the range of gravity may be obtained from the difference between the derived abundances of $\mathrm{Fe}$ II and $\mathrm{Fe}$ I. This difference (see Table 6) is $[\mathrm{Fe} \mathrm{II} / \mathrm{H}]-[\mathrm{Fe} \mathrm{I} / \mathrm{H}]=0.05 \pm 0.12$, where the uncertainty is the quadratic sum of $\sigma$ of the measured Fe II abundance (that of Fe I, being 0.02 dex, is negligible when added in quadrature) and of the error on the Fe I abundance arising from uncertainties in the temperature (see Table 5 of Paper II and the discussion in $\S 4.2$ ). Thus, the maximum difference between the abundances of the ionized and neutral iron species, at a $2 \sigma$ confidence level, is $\left([\mathrm{Fe} / \mathrm{H}] \mathrm{II}-[\mathrm{Fe} / \mathrm{H}]_{\mathrm{II}}\right)_{\max }=0.28$. According to the sensitivity of the abundances to the atmospheric parameters given in Table 5 of Paper II, this corresponds to a lower limit for the gravity of $\log g=3.7$ dex.

Taking into account this constraint, the probability for masses larger than $0.95 M_{\odot}$ is reduced to about 0.003 (since only the low-luminosity phase is acceptable for larger masses). Thus, on the basis of these simple statistical considerations, it is reasonable to assume that the mass of the primary lies in the range $0.75<M / M_{\odot}<0.95$.

\subsubsection{Secondary Star Mass}

It is very likely that the secondary star has undergone thermal pulses during its AGB phase, as we observe the expected nucleosynthesis products (see $\S 4.2$ and following): the $s$-process elements that we detect in the primary star must have been produced in the secondary, as the primary seems to be presently on the main sequence. The AGB nucleosynthesis-enriched material was then transferred to the primary star, polluting its atmosphere. The minimum amount of material required to create the signatures characteristic of CH stars is quite small, as the polluted star is very metal-poor.

The mass of the secondary, which is now likely a white dwarf, is likely to be consistent with the mass of the degenerate $\mathrm{CO}$ core of the star in its AGB phase. Thus, based on this assumption, it is possible to obtain a reliable lower limit to the mass of this star. The minimum core mass for a star to experience third dredge-up is a (theoretically) very wellstudied quantity over the metallicity range $-1.3<$ $[\mathrm{Fe} / \mathrm{H}]<0$ (Herwig et al. 1997; Boothroyd \& Sackman 1988a, 1988b, 1988c, 1988d; Marigo, Girardi, \& Bressan 1999). However, no detailed computation has been performed for metallicities as low as that of HE 0024-2523, hence extrapolation to such low metallicities might be premature.

Since we seek an upper limit for the value of $\sin i$, high precision is not crucial; a reasonable estimate of this quantity can provide a meaningful constraint. Hence we adopt the value of $0.6 M_{\odot}$ from an ad hoc computation (O. Straniero 2002, private communication).

\subsubsection{Orbital Inclination and Synchronous Rotation}

By inversion of the expression for the mass function, $f(m)=m_{2}^{3} \sin ^{3} i /\left(m_{1}+m_{2}\right)^{2}$ (which is known from the orbital solution), and substitution for $m_{1}$ and $m_{2}$, respectively, of the upper and lower limits estimated in the previous sections, we obtain

$$
\sin i \leq \sqrt[3]{\frac{\left(m_{1}+m_{2}\right)^{2}}{m_{2}^{3}} f(m)}=0.82,
$$


which corresponds to an inclination of $i \leq 55^{\circ} .1$. It should be kept in mind that this is an upper limit to the angle and not an estimate of the angle itself. It is noteworthy that the value of $\sin i$ is far more sensitive to the mass of the secondary than it is to the primary.

On the basis of this upper limit on the orbital inclination angle, we can derive an upper limit to the value of the synchronous rotational velocity. A star is in a synchronous orbit if its rotational period is the same as its period of revolution. Since the orbital period has been measured, by using the derived upper limit on $\sin i$ for the upper limit of the projected value of the rotational velocity we obtain

$$
v_{\text {rot,syn }} \sin i \leq \frac{2 \pi \mathrm{R} \sin i}{P},
$$

where $v_{\text {rot,syn }}$ is the upper limit of the value of the synchronous rotational velocity, $R$ is the radius of the star, and $P$ is the orbital revolution period. Using the same $Y^{2}$ (Yi et al. 2001) isochrone as that described in Paper I, the value for the radius corresponding to the atmospheric parameters adopted was computed to be $R=1.10 R_{\odot}$. Substituting this value in equation (3), we obtain an upper limit for the synchronous projected rotational velocity of $v_{\text {rot,syn }} \sin i \leq 13.5 \pm 3.9 \mathrm{~km} \mathrm{~s}^{-1}$. The uncertainty is mainly due to the errors in the determination of the radius, since the period and the mass function are determined with a much higher degree of accuracy. The error of the radius depends on the uncertainties of the effective temperature and gravity. Such uncertainties are, respectively, about $\pm 100 \mathrm{~K}$ in $T_{\text {eff }}$ (which translates into an uncertainty of $10 \%$ on the radius) and, as discussed in $\S 3.3 .1, \pm 0.3 \mathrm{dex}$ in $\log g$ (about $25 \%$ in the radius). The total error on the radius, and consequently on the $v_{\text {rot.syn }} \sin i$, is $29 \%(1 \sigma)$.

The measured value of $v_{\text {rot,syn }} \sin i=9.7 \pm 1.5 \mathrm{~km} \mathrm{~s}^{-1}$ is compatible with the computed upper limit. Since the value of the orbital semimajor axis is only a few times larger than the inferred radius, it is likely that tidal forces led the system to reach a state of synchronicity and a circular orbit, the latter of which has been derived from our observations. Thus the orbit can be considered synchronous and the observed $v_{\text {rot }} \sin i$ the true synchronous rotational velocity of the primary.

Assuming synchronicity, we can derive the value of $\sin i$ and hence a relationship between the values of the masses of the two components. This value is subject to the error computed for the upper limit of the angle (see eq. [2]), plus that due to that of the measured rotational velocity, which is $\pm 15 \%$. In this manner, using equation (3) and substituting $v_{\text {rot }} \sin i$ for $v_{\text {rot,syn }} \sin i$, we obtain $\sin i=0.59 \pm 0.19$, corresponding to an angle of $i=\left(36_{-7}^{+15}\right) \mathrm{deg}$.

The expression of the mass function links the values of the primary and the secondary masses, so that once one of them is known, under the hypothesis of a synchronous orbit, the other is uniquely determined. However, in this case this computation does not add any information, as the mass range of the secondary corresponding to that computed for the primary in $\$ 3.3 .1-$ calculated from the mass function using for $\sin i$ the maximum, minimum, and mean value allowed in the case of synchronous orbit - covers the entire interval from $0.6 M_{\odot}$ to (and beyond) the Chandrasekar limit (see Table 4). Thus, the only limits we are able to derive
TABLE 4

Mass of the Secondary as a Function of the Mass of THE PRIMARY

\begin{tabular}{|c|c|c|c|c|c|}
\hline \multicolumn{2}{|c|}{$\sin i=0.59$} & \multicolumn{2}{|c|}{$\sin i_{\min }=0.40$} & \multicolumn{2}{|c|}{$\sin i_{\max }=0.78$} \\
\hline $\begin{array}{c}m_{1} \\
\left(M_{\odot}\right)\end{array}$ & $\begin{array}{c}m_{2} \\
\left(M_{\odot}\right)\end{array}$ & $\begin{array}{c}m_{1} \\
\left(M_{\odot}\right)\end{array}$ & $\begin{array}{c}m_{2} \\
\left(M_{\odot}\right)\end{array}$ & $\begin{array}{c}m_{1} \\
\left(M_{\odot}\right)\end{array}$ & $\begin{array}{c}m_{2} \\
\left(M_{\odot}\right)\end{array}$ \\
\hline 0.60. & 0.81 & 0.60 & 1.62 & 0.60 & 0.53 \\
\hline $0.65 \ldots \ldots .$. & 0.83 & 0.65 & 1.66 & 0.65 & 0.55 \\
\hline $0.70 \ldots \ldots$. & 0.85 & 0.70 & 1.70 & 0.70 & 0.56 \\
\hline $0.75 \ldots \ldots$. & 0.87 & 0.75 & 1.74 & 0.75 & 0.58 \\
\hline $0.80 \ldots \ldots$. & 0.90 & 0.80 & 1.77 & 0.80 & 0.59 \\
\hline $0.85 \ldots \ldots$. & 0.92 & 0.85 & 1.81 & 0.85 & 0.60 \\
\hline $0.90 \ldots \ldots .$. & 0.94 & 0.90 & 1.84 & 0.90 & 0.62 \\
\hline $0.95 \ldots \ldots .$. & 0.95 & 0.95 & 1.88 & 0.95 & 0.63 \\
\hline $1.00 \ldots \ldots$. & 0.97 & 1.00 & 1.91 & 1.00 & 0.64 \\
\hline $1.05 \ldots \ldots$. & 0.99 & 1.05 & 1.94 & 1.05 & 0.65 \\
\hline $1.10 \ldots \ldots$ & 1.01 & 1.10 & 1.97 & 1.10 & 0.67 \\
\hline $1.15 \ldots \ldots \ldots$ & 1.03 & 1.15 & 2.00 & 1.15 & 0.68 \\
\hline
\end{tabular}

are $0.75 M_{\odot}<m_{1}<0.95 \quad M_{\odot}$ and $0.60 \quad M_{\odot}<m_{2}<$ $1.4 M_{\odot}$.

\section{CHEMICAL COMPOSITION}

Paper II described the results for the chemical composition of HE 0024-2523 from the HIRES spectra taken in 2000 September. In the following sections we present the results obtained from the abundance analysis from line equivalent widths ( $(4.2)$ and spectral syntheses $(\S 4.3)$ for both the UVES and HIRES spectra. For both purposes we used the UVES spectrum with the highest signal-to-noise ratio (i.e., that taken on 2001 August 27) and the sum of the HIRES spectra obtained in 2000 September. The addition of the UVES spectrum to our data sample improves the signal-to-noise ratio and permits the detection and measurement of lines that were not within the spectral range covered by the HIRES spectra.

\subsection{Atmospheric Parameters}

The procedure used to determine the atmospheric parameters for HE 0024-2523 have been thoroughly explained in Paper I: $T_{\text {eff }}$ is derived from broadband colors, taking the mean of the dereddened colors $V-K$ and $V-J$; once $T_{\text {eff }}$ is specified, $\log g$ is obtained from the $\mathrm{Y}^{2}$ isochrones.

With $T_{\text {eff }}$ and $\log g$ fixed, $[\mathrm{Fe} / \mathrm{H}]$ is determined interactively by matching the observed equivalent widths (EWs) to those computed by integrating the line profiles of the adopted atmospheric model, obtained from the equation of transport computed at different wavelengths along each line. The model atmosphere we adopt was extracted by interpolation from the Kurucz grid of models (Kurucz 1995a) without overshooting. This model assumes a scaled solar composition: while this is not strictly appropriate in the case of HE 0024-2523, modifications in the model structure are not very large. A crude estimate of these modifications to the model atmosphere due to the high carbon abundance $([\mathrm{C} / \mathrm{Fe}]=+2.6)$ can be obtained with the following method. We measured the integrated absorption due to all the spectral lines in the range between 4300 and 4335 $\AA$, both in the program star and in another star of the Keck Pilot Program (HE 2344-2800), which has almost identical atmospheric parameters. This interval was chosen as it is 
one of the spectral ranges most affected by the presence of $\mathrm{CH}$. The resulting ratio of the summed absorption integrated over this spectral range is $\sim 2.5$. As a very conservative estimate, ${ }^{12}$ we assumed that the $\mathrm{CH}$ absorption affects the model in the same way as a spectral interval, which accounts for 10 times $(\sim 0.05$ of the flux, obtained from the integration of the equation of the flux of a blackbody at the same temperature as HE 0024-2523) as much flux as that emitted in the range 4300 to $4335 \AA$ ( $\sim 0.005$ of the flux). On this basis, i.e., that the remaining spectral regions accounting for about $95 \%$ of the flux are negligibly affected, we deduced that the difference in the flux of the star with $[\mathrm{C} / \mathrm{H}]=+2.6$, with respect to that with scaled solar carbon abundance, is $\sim 7.5 \%$. At a metallicity of $[\mathrm{Fe} / \mathrm{H}]=-2.7$, this translates into an error of 0.03 dex, which is negligible compared with the errors of the abundances due to the uncertainties in the atmospheric parameters themselves. ${ }^{13}$

Equivalent widths for the UVES spectrum were measured in the same way as described in Paper II for the HIRES spectra. Since the resolution of the UVES spectrum is almost identical to that of the HIRES one, the signal-tonoise ratio is only slightly better, and the procedure used for the measurements and selection of EWs are identical, we expect the internal errors to be similar to those evaluated in Paper II. The same follows for the external errors (see $\S 3$ of Paper II). A comparison between the EWs obtained from the UVES spectrum and those from the HIRES spectra showed that the EWs from the VLT spectra are systematically larger than those measured from the Keck spectra, as can be seen in Figure 3 (top). Figure 3 (bottom) shows the UVES EWs versus those obtained from HIRES; the upper line is described by the equation $\mathrm{EW}_{\mathrm{H}}=\mathrm{EW}_{\mathrm{U}}$, while the lower line is the best-fit relation, described by the equation

$$
\mathrm{EW}_{\mathrm{H}}=(0.82 \pm 0.04) \mathrm{EW}_{\mathrm{U}}+(1.56 \pm 2.10) \mathrm{m} \AA .
$$

The rms scatter around the regression line is $6.8 \mathrm{~m} \AA$; assuming that both sets have equal errors, we can estimate a typical error of $4.8 \mathrm{~m} \AA$ for our measurements in a single spectrum. This difference in the EWs does not depend on the measurement procedure or on the continuum tracing. To show this, we compared several strong lines by superposing some sample regions from the two spectra in Figure 4; the difference between the two spectra is directly visible there.

A contribution due to inadequate subtraction of scattered light during the reduction does not seem likely. In the UVES spectra, the $\mathrm{O}_{2}$ telluric lines around $7630 \AA$ reach down to zero intensity, indicating a proper treatment of scattered light. Furthermore, the comparison between the EWs for HD 140283 from an HIRES spectrum and those from the literature (see Paper II) showed no systematic difference, suggesting that, at least in this case, scattered light was subtracted accurately. We have no explanation at present for this difference, although it is noteworthy that the spectra were taken at different phases (spaced roughly by a quarter of an orbit).

12 Even after having taken into account the contribution due to other $\mathrm{CH}$ bands, $\mathrm{CN}$ bands and $\mathrm{CO}$ bands, this estimate is essentially an upper limit. This spectral range is one of the most affected by a high carbon abundance since it covers most of the $G$ band of $\mathrm{CH}$ absorption. A more realistic value would be $\sim 2$.

${ }^{13}$ Using a different test, Aoki et al. 2003 found that the enhancements in $\mathrm{C}$ and $\mathrm{N}$ have only a small effect on the model atmospheres in the temperature range $4000-6000 \mathrm{~K}$. For an actual calculation of the effects on C and N excesses on the atmospheric structure we refer the reader Hill et al. 2000.

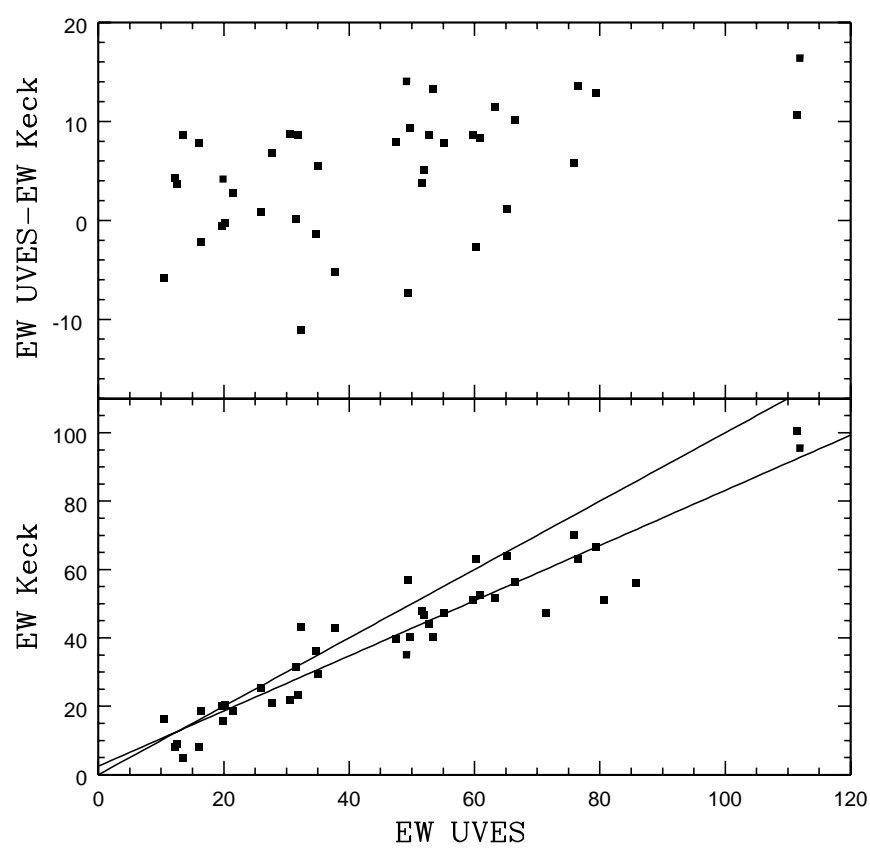

FIG. 3.-Comparison between the EWs measured from the UVES spectrum with those measured from the HIRES 2000 September spectra. Bottom: UVES EWs vs. those of HIRES. The upper line is described by the equation $\mathrm{EW}_{\mathrm{H}}=\mathrm{EW}_{\mathrm{U}}$, while the lower line is the best fit and its equation is $\mathrm{EW}_{\mathrm{H}}=(0.82 \pm 0.04) \mathrm{EW}_{\mathrm{U}}+(1.56 \pm 2.10)$; the rms of the fit is $6.8 \mathrm{~m} \AA$. Top: Difference between the corresponding EWs and the UVES EWs.

Since not all the lines were detected and measured in both spectra, to calculate the EWs to be used for the analysis we computed an "average" correction, i.e., two equations that allow the calculation of the expected mean value of the EWs from the value measured in only one of the spectra. The coefficients of the two equations were calculated on the basis of the lines detected in both spectra and assuming that the expected value of the mean EW depends linearly on EWs measured in only one of the spectra. We obtain

$$
\begin{aligned}
& \mathrm{EW}_{\text {mean }}=(1.05 \pm 0.03) \mathrm{EW}_{\mathrm{H}}+(1.41 \pm 1.21) \mathrm{m \AA}, \\
& \mathrm{EW}_{\text {mean }}=(0.91 \pm 0.02) \mathrm{EW}_{\mathrm{U}}+(0.78 \pm 1.06) \mathrm{m} \AA .
\end{aligned}
$$

Thus, in the abundance analysis we used the mean of the EWs when a line was detected and measured in both spectra or a value corrected using equation (5) or (6) if the EW was measured, respectively, only in the HIRES or the UVES spectrum.

The microturbulent velocity was derived by elimination of any trend in abundances derived from $\mathrm{Fe}$ I lines with the expected EW. The final value adopted herein turned out to be different from that of Paper I, as more lines from UVES spectra were added. In the present analysis, we find $T_{\text {eff }}=6625 \mathrm{~K}, \quad[\mathrm{Fe} / \mathrm{H}]=-2.72, \log g=4.3$ dex, and $v_{\text {micro }}=1.4 \mathrm{~km} \mathrm{~s}^{-1}$, compared with $[\mathrm{Fe} / \mathrm{H}]=-2.65$ and $v_{\text {micro }}=0.58 \mathrm{~km} \mathrm{~s}^{-1}$ in Paper II. The list of EWs, along with the excitation potential and assumed $\log g f$ of the lines is provided in Table 5 .

\subsection{Abundance Analysis}

The abundance analysis was performed using the values of the EWs ${ }^{14}$ described in the previous section and listed in

\footnotetext{
${ }^{14}$ For the VALD database see http://www.astro.univie.ac.at/ vald/.
} 

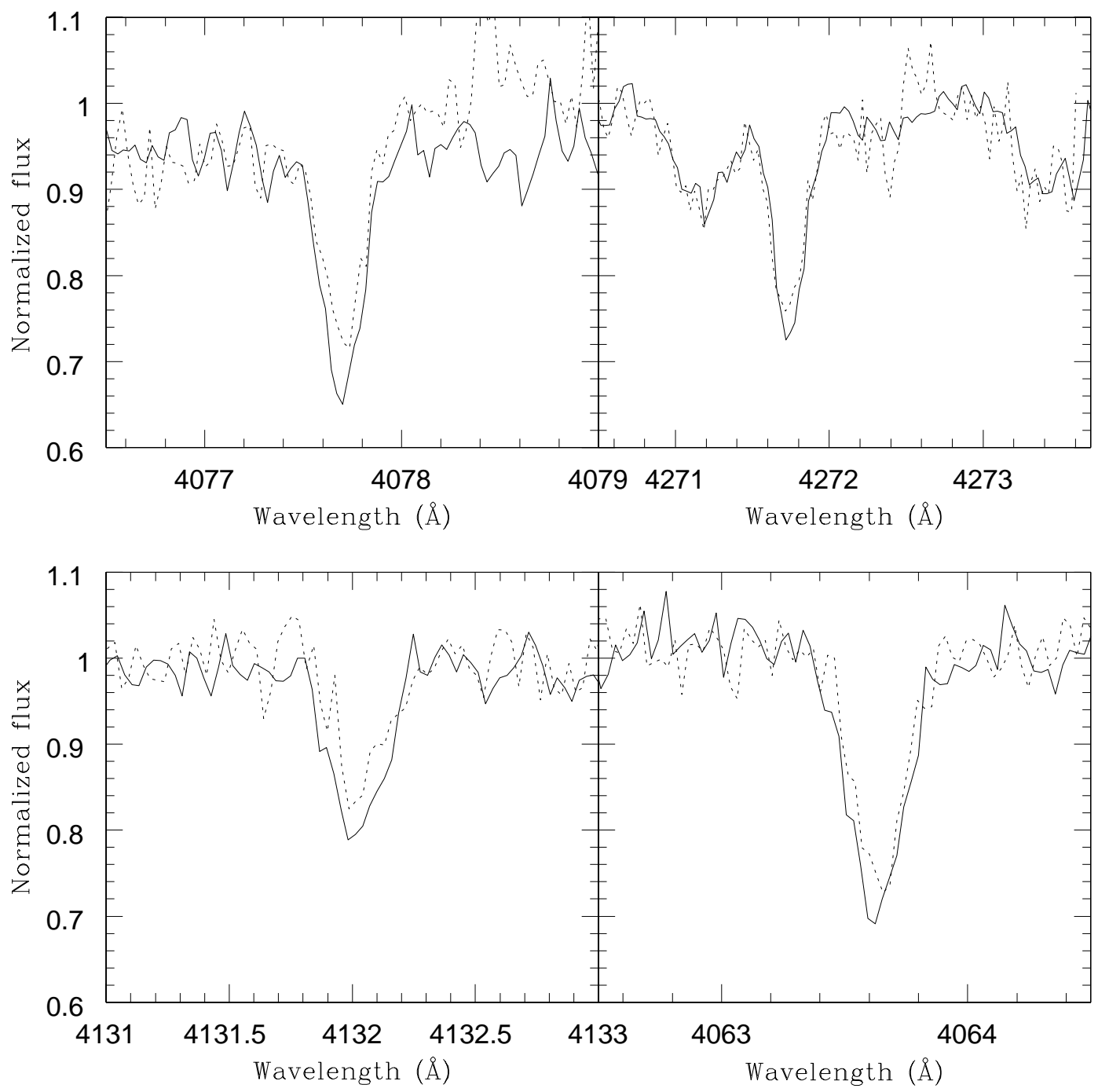

FIG. 4.-Comparison between four spectral regions from the UVES spectrum (solid line) and HIRES 2000 September spectra (dashed line). Note that the lines appear slightly stronger in the UVES spectra.

Table 5. The resulting abundance and element ratios for each species are listed in Table 6, along with the number of lines used in the abundance analysis from equivalent width measurements of a given ion and $\sigma$, the rms scatter in abundance obtained from individual lines within the set of lines used for that particular ion. The abundances of neutral species are computed with respect to $\mathrm{Fe}$ I, while singly ionized species are compared with $\mathrm{Fe}$ II, to minimize the effect due to the choice of atmospheric parameters.

In Paper II we evaluated the sensitivity of the derived abundances to the atmospheric parameters for the method used, and the results are displayed in Table 6 of that paper. Since the uncertainties on the atmospheric parameters and the method used for the abundance analysis are the same, these results still hold for HE 0024-2523. The cumulative errors, which take into account the scatter in the abundances derived from the lines and the effect of the uncertainties on the atmospheric parameters, are given in column (5) of Table 6.

\subsubsection{Abundances}

The solar abundances we have assumed to compute the abundance ratios are the same as in Paper II, i.e., those obtained from the analysis of the solar spectrum using
Kurucz's models (1995a). These results are very similar to those of Anders \& Grevesse 1989, with the exception of iron, which is lower by about 0.1 dex than their photospheric abundance value, while it roughly agrees with their meteoritic value. The results obtained from the abundance analysis (see Table 6) are quite similar to those presented in Paper II. The detection of the $\mathrm{Zr}$ II line at $4149.2 \AA$ and of the $\mathrm{Y}$ II line at $4883.7 \AA$ are noteworthy additions. Since the signal-tonoise ratio in those regions was not very high and we did not detect any other lines of these elements, even if other lines of similar strength are expected to be detectable, we considered those measured EWs as upper limits. The difference between the measured abundances of Ti I and Ti II might suggest that the assumed $T_{\text {eff }}$ is too high; however, the $\mathrm{Fe}_{\mathrm{I}}$ and $\mathrm{Fe}$ II abundances are very similar and seem to invalidate this hypothesis. On the other hand, the three Ti I lines detected are very weak and the abundances derived from them should be attributed low weight.

Paper II provides a thorough and detailed description of the sources of uncertainties in the derived abundance for $\mathrm{Fe}$, describes the oscillator strengths assumed, and provides a comparison with a purely spectroscopic analysis. A similar discussion is also provided for the $\alpha$-elements; we refer the interested reader to $\S \S 4.1$ and those that follow in the afore- 
TABLE 5

EQuivalent WidThS FOR HE 0024-2523

\begin{tabular}{|c|c|c|c|c|c|c|}
\hline Ion & $\begin{array}{l}\lambda \\
(\AA)\end{array}$ & $\begin{array}{c}\chi \\
(\mathrm{eV})\end{array}$ & $\log g f$ & $\begin{array}{l}\text { Reference for } \\
\quad \log g f\end{array}$ & $\begin{array}{l}\text { EW } \\
(\mathrm{m} \AA)\end{array}$ & Weight \\
\hline OІ.............. & 7771.95 & 9.11 & 0.33 & 1 & 8.7 & 1 \\
\hline $\mathrm{Na} \mathrm{I}_{\ldots} . . . .$. & 5889.95 & 0.00 & 0.10 & 2 & 71.2 & 1 \\
\hline $\mathrm{Na} I \ldots \ldots . .$. & 5895.92 & 0.00 & -0.20 & 2 & 45.6 & 1 \\
\hline $\operatorname{Mg}{ }_{I} \ldots \ldots$ & 4057.52 & 4.34 & -1.20 & 3 & 13.3 & 1 \\
\hline $\operatorname{Mg}_{I} \ldots \ldots$ & 4167.28 & 4.34 & -1.00 & 3 & 10.8 & 0 \\
\hline $\operatorname{Mg}_{I} \ldots \ldots$ & 4703.00 & 4.34 & -0.67 & 3 & 19.9 & 1 \\
\hline $\operatorname{Mg}_{I} \ldots . .$. & 5172.70 & 2.71 & -0.38 & 3 & 121.4 & 1 \\
\hline $\operatorname{Mg}_{I} \ldots \ldots$ & 5183.62 & 2.72 & -0.16 & 3 & 142.7 & 1 \\
\hline 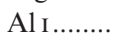 & 3961.52 & 0.00 & -0.34 & 4 & 49.3 & 1 \\
\hline Si I.......... & 3905.53 & 1.91 & -1.04 & 5 & 83.8 & 1 \\
\hline Ca I ........ & 4226.74 & 0.00 & 0.24 & 6 & 103.7 & 1 \\
\hline Ca I ........ & 4425.44 & 1.88 & -0.36 & 6 & 13.6 & 1 \\
\hline Ca I ........ & 4435.69 & 1.89 & -0.52 & 6 & 8.6 & 1 \\
\hline Ca I ........ & 4454.79 & 1.90 & 0.26 & 4 & 25.6 & 1 \\
\hline Sc II ........ & 4246.82 & 0.32 & 0.32 & 7 & 32.2 & 1 \\
\hline Sc II ........ & 4314.08 & 0.62 & -0.10 & 7 & 15.5 & 1 \\
\hline Sc II ........ & 4320.73 & 0.60 & -0.26 & 7 & 12.3 & 1 \\
\hline Ti I ......... & 3958.22 & 0.05 & -0.16 & 8 & 10.7 & 1 \\
\hline Ti I ......... & 3998.64 & 0.05 & -0.05 & 8 & 11.3 & 1 \\
\hline Ti I ......... & 4533.25 & 0.85 & 0.48 & 9 & 6.4 & 1 \\
\hline Ti II ........ & 3900.54 & 1.13 & -0.45 & 7 & 39.8 & 1 \\
\hline Ti II ........ & 4012.39 & 0.57 & -1.61 & 7 & 13.8 & 1 \\
\hline Ti II ........ & 4028.35 & 1.89 & -0.87 & 10 & 9.2 & 0 \\
\hline Ti II ........ & 4301.93 & 1.16 & -1.20 & 11 & 11.1 & 1 \\
\hline Ti II ........ & 4395.03 & 1.08 & -0.51 & 11 & 35.4 & 1 \\
\hline Ti II ........ & 4399.77 & 1.24 & -1.29 & 10 & 12.1 & 0 \\
\hline Ti II ........ & 4417.72 & 1.16 & -1.16 & 10 & 10.1 & 1 \\
\hline Ti II ........ & 4443.81 & 1.08 & -0.70 & 11 & 26.4 & 1 \\
\hline Ti II ........ & 4468.51 & 1.13 & -0.60 & 11 & 31.4 & 1 \\
\hline Ti II ........ & 4501.28 & 1.12 & -0.76 & 11 & 25.3 & 1 \\
\hline Ti II ........ & 4533.97 & 1.24 & -0.64 & 10 & 27.6 & 1 \\
\hline Ti II ........ & 4549.64 & 1.58 & -0.47 & 10 & 31.1 & 0 \\
\hline Ti II ........ & 4563.77 & 1.22 & -0.82 & 10 & 20.0 & 1 \\
\hline Ti II ........ & 4571.98 & 1.57 & -0.34 & 10 & 26.3 & 1 \\
\hline Ti II ......... & 4589.95 & 1.24 & -1.65 & 10 & 5.2 & 1 \\
\hline Cr I................. & 4254.33 & 0.00 & -0.11 & 12 & 13.3 & 1 \\
\hline Cri................. & 4274.79 & 0.00 & -0.23 & 12 & 18.4 & 1 \\
\hline Mn I...... & 4030.75 & 0.00 & -0.47 & 13 & 23.4 & 1 \\
\hline Mn I ....... & 4033.06 & 0.00 & -0.62 & 13 & 14.7 & 1 \\
\hline Fe I......... & 3865.52 & 1.01 & -0.98 & 14 & 48.5 & 1 \\
\hline $\mathrm{Fe}_{\mathrm{I}} \ldots \ldots \ldots$ & 3886.29 & 0.05 & -1.08 & 15 & 70.9 & 1 \\
\hline $\mathrm{Fe}_{\mathrm{I}} \ldots \ldots \ldots$ & 3887.06 & 0.91 & -1.14 & 14 & 42.8 & 1 \\
\hline Fe I.......... & 3895.67 & 0.11 & -1.67 & 15 & 51.2 & 1 \\
\hline $\mathrm{Fe}_{\mathrm{I}} \ldots \ldots \ldots$ & 3899.72 & 0.09 & -1.53 & 15 & 46.8 & 1 \\
\hline Fe I......... & 3902.96 & 1.56 & -0.47 & 16 & 49.7 & 1 \\
\hline Fe I.............. & 3906.49 & 0.11 & -2.24 & 15 & 27.5 & 1 \\
\hline $\mathrm{Fe}_{\mathrm{I}} \ldots \ldots \ldots$ & 3920.27 & 0.12 & -1.75 & 15 & 55.5 & 1 \\
\hline Fe I.......... & 3922.92 & 0.05 & -1.65 & 15 & 56.7 & 1 \\
\hline Fe I................. & 3930.31 & 0.09 & -1.59 & 7 & 57.5 & 1 \\
\hline $\mathrm{Fe}_{\mathrm{I}} \ldots \ldots \ldots$ & 4005.24 & 1.56 & -0.61 & 16 & 45.0 & 1 \\
\hline Fe I.......... & 4045.81 & 1.49 & 0.28 & 16 & 76.5 & 1 \\
\hline Fe I......... & 4063.59 & 1.56 & 0.08 & 17 & 73.0 & 1 \\
\hline $\mathrm{Fe}_{\mathrm{I}} \ldots \ldots \ldots$ & 4071.74 & 1.61 & -0.02 & 16 & 61.5 & 1 \\
\hline Fe I......... & 4118.56 & 3.57 & 0.14 & 17 & 15.7 & 0 \\
\hline Fe I.......... & 4132.06 & 1.61 & -0.82 & 17 & 42.1 & 1 \\
\hline $\mathrm{Fe}_{\mathrm{I}} \ldots \ldots \ldots$ & 4143.87 & 1.56 & -0.62 & 17 & 43.6 & 1 \\
\hline Fe I......... & 4187.05 & 2.45 & -0.55 & 18 & 12.0 & 1 \\
\hline Fe I.......... & 4187.81 & 2.43 & -0.55 & 18 & 16.5 & 1 \\
\hline Fe I............ & 4198.33 & 2.40 & -0.72 & 18 & 17.8 & 0 \\
\hline Fe I................. & 4199.10 & 3.05 & 0.16 & 17 & 20.4 & 1 \\
\hline Fe I......... & 4202.04 & 1.49 & -0.71 & 16 & 37.7 & 1 \\
\hline Fe I......... & 4227.44 & 3.33 & 0.27 & 17 & 11.2 & 1 \\
\hline Fe I.......... & 4235.95 & 2.43 & -0.34 & 18 & 27.4 & 1 \\
\hline Fe I.......... & 4250.13 & 2.47 & -0.41 & 18 & 12.9 & 1 \\
\hline
\end{tabular}

TABLE 5-Continued

\begin{tabular}{|c|c|c|c|c|c|c|}
\hline Ion & $\begin{array}{c}\lambda \\
(\AA)\end{array}$ & $\begin{array}{c}\chi \\
(\mathrm{eV})\end{array}$ & $\log g f$ & $\begin{array}{l}\text { Reference for } \\
\qquad \log g f\end{array}$ & $\begin{array}{c}\text { EW } \\
(\mathrm{m} \AA)\end{array}$ & Weight \\
\hline Fe I......... & 4250.80 & 1.56 & -0.38 & 17 & 30.9 & 0 \\
\hline Fe I............. & 4260.49 & 2.40 & 0.14 & 19 & 37.5 & 1 \\
\hline Fe I......... & 4271.16 & 2.45 & -0.35 & 18 & 24.2 & 1 \\
\hline Fe ${ }_{1} \ldots \ldots . .$. & 4271.77 & 1.49 & -0.16 & 16 & 59.4 & 1 \\
\hline Fe I......... & 4282.41 & 2.18 & -0.78 & 17 & 10.3 & 1 \\
\hline Fe I......... & 4294.14 & 1.49 & -0.97 & 20 & 36.6 & 1 \\
\hline Fe I.......... & 4299.25 & 2.43 & -0.35 & 19 & 23.5 & 1 \\
\hline Fe ${ }_{1} \ldots \ldots . .$. & 4307.91 & 1.56 & -0.07 & 17 & 65.9 & 1 \\
\hline Fe I............ & 4325.77 & 1.61 & 0.01 & 17 & 64.6 & 1 \\
\hline Fe I......... & 4383.56 & 1.49 & 0.20 & 16 & 73.0 & 1 \\
\hline Fe I......... & 4404.76 & 1.56 & -0.14 & 16 & 61.7 & 1 \\
\hline Fe I......... & 4415.13 & 1.61 & -0.62 & 16 & 40.3 & 1 \\
\hline Fe I......... & 4920.51 & 2.83 & 0.15 & 17 & 23.7 & 1 \\
\hline Fe I......... & 4957.61 & 2.81 & 0.23 & 19 & 40.4 & 1 \\
\hline Fe I......... & 5269.55 & 0.86 & -1.32 & 14 & 52.8 & 0 \\
\hline Fe II........ & 4233.17 & 2.57 & -2.00 & 7 & 17.4 & 1 \\
\hline Fe II........ & 4583.84 & 2.81 & -2.02 & 7 & 17.2 & 1 \\
\hline Fe II........ & 4923.93 & 2.88 & -1.32 & 7 & 18.3 & 1 \\
\hline Fe II........ & 5018.45 & 2.89 & -1.22 & 7 & 39.3 & 1 \\
\hline Sr II ........ & 4077.71 & 0.00 & 0.15 & 4 & 69.7 & 1 \\
\hline Sr II ......... & 4215.52 & 0.00 & -0.17 & 4 & 53.1 & 1 \\
\hline Y II ......... & 4883.69 & 1.08 & 0.07 & 20 & $<5.9$ & 1 \\
\hline Zr II........ & 4149.20 & 0.80 & -0.03 & 14 & $<9.0$ & 1 \\
\hline Ba II ....... & 4554.04 & 0.00 & 0.17 & 21 & 106.1 & 1 \\
\hline Ba II ....... & 6141.75 & 0.70 & 0.00 & 21 & 56.5 & 1 \\
\hline Ba II ....... & 6496.91 & 0.60 & -0.38 & 21 & 34.2 & 1 \\
\hline
\end{tabular}

${ }^{a}$ Weight attributed to the line; lines outside a $3 \sigma$ interval were given zero weight.

REFERENCES.-(1) Lambert 1978; (2) Biemont et al. 1991; (3) VALD database (as of 2001 August); (4) Wiese \& Martin 1980; (5) O'Brian \& Lawler 1991a; (6) Smith \& Raggett 1981; (7) Kurucz 1995b; (8) Blackwell et al. 1982b; (9) Blackwell et al. 1982a; (10) Magain 1985; (11) Bizzarri et al. 1993; (12) Blackwell, Menon, \& Petford; (13) Blackwell et al. 1979a; (14) Hannaford et al. 1982; (15) Blackwell, Petford, \& Shallis 1979b; (16) Blackwell et al. 1982c; (17) O'Brian et al. 1991; (18) Blackwell et al. 1980; (19) Bard \& Kock 1994; (20) Biémont et al. 1981; (21) Holweger \& Müller 1974.

mentioned paper for a complete discussion of these aspects of the analysis. A basic assumption in our procedure is that departures from LTE are negligible for Fe. While there is no doubt that Fe II lines form very close to LTE, Thévenin \& Idiart (1999) proposed that there is a significant overionization of Fe in metal-poor stars. According to their predictions, an LTE analysis should underestimate the abundances derived from $\mathrm{Fe}$ I lines by about 0.3 dex in stars with atmospheric parameters similar to HE 0024-2523. While such an error would have no major impact on the conclusions of this paper, we remark that there is no evidence for such a large error in the $\mathrm{Fe}$ ionization equilibrium from our data (this difference would correspond to an error of about 1.2 dex in the surface gravity, much larger than that compatible with the location of the star, which is close to the turnoff). On the other side, the prediction by Thévenin \& Idiart is not confirmed by other statistical equilibrium computations. Gratton et al. (1999) estimated a much smaller nonLTE correction of about 0.1 dex for stars with atmospheric parameters similar to HE 0024-2523, but they also noted that this value has to be considered an upper limit to real non-LTE corrections. The result by Gratton et al. is based on a comparison of expected departures from LTE with empirical determinations of $\mathrm{Fe}$ ionization equilibrium for stars in which departures from LTE are expected to be much 
TABLE 6

Abundance Summary FOR HE 0024-2523

\begin{tabular}{|c|c|c|c|c|c|}
\hline Element Ratio & $n^{\mathrm{a}}$ & $\begin{array}{l}\text { Abundance } \\
\text { (dex) }\end{array}$ & $\begin{array}{l}\text { rms } \\
\text { (dex) }\end{array}$ & $\begin{array}{l}\text { Error } \\
(\text { dex })\end{array}$ & Method \\
\hline$\left[\mathrm{Fe} \mathrm{I}_{\mathrm{I}} / \mathrm{H}\right] \ldots$. & 36 & -2.72 & 0.12 & 0.12 & EWs \\
\hline$[$ FeII $/ \mathrm{H}] \ldots \ldots \ldots \ldots$ & 3 & -2.67 & 0.14 & 0.14 & EWs \\
\hline $\log \epsilon(\mathrm{Li}) \ldots \ldots \ldots \ldots$ & & 1.5 & $\ldots$ & 0.1 & Syn \\
\hline$[\mathrm{C} / \mathrm{Fe}] \mathrm{I} \ldots \ldots \ldots \ldots . . . .$. & & 2.6 & $\ldots$ & 0.1 & Syn \\
\hline$[\mathrm{N} / \mathrm{Fe}] \mathrm{I} \ldots \ldots \ldots \ldots$ & & 2.1 & $\ldots$ & 0.1 & Syn \\
\hline$[\mathrm{O} / \mathrm{Fe}] \mathrm{I} \ldots \ldots \ldots \ldots . . . .$. & 1 & $0.40^{\mathrm{b}}$ & $\ldots$ & $\ldots$ & EWs \\
\hline$[\mathrm{Na} / \mathrm{Fe}] \mathrm{I} \ldots \ldots \ldots \ldots$ & 2 & $-0.17^{b}$ & 0.13 & 0.13 & EWs \\
\hline$[\mathrm{Mg} / \mathrm{Fe}] \mathrm{I} \ldots \ldots \ldots \ldots$ & 4 & 0.73 & 0.11 & 0.06 & EWs \\
\hline$[\mathrm{Al} / \mathrm{Fe}] \mathrm{I} . . . \ldots \ldots \ldots . . .$. & 1 & -0.35 & $\ldots$ & $\ldots$ & EWs \\
\hline$[\mathrm{Si} / \mathrm{Fe}] \mathrm{I} \ldots \ldots \ldots \ldots$ & 1 & 0.34 & $\ldots$ & $\ldots$ & EWs \\
\hline$[\mathrm{Ca} / \mathrm{Fe}] \mathrm{I} \ldots \ldots \ldots . . .$. & 4 & 0.66 & 0.13 & 0.17 & EWs \\
\hline$[\mathrm{Sc} / \mathrm{Fe}] \mathrm{II} \ldots \ldots \ldots \ldots . . .$. & 3 & 0.37 & 0.14 & 0.14 & EWs \\
\hline$[\mathrm{Ti} / \mathrm{Fe}] \mathrm{I} \ldots \ldots \ldots \ldots$ & 3 & 0.85 & 0.13 & 0.10 & EWs \\
\hline$[\mathrm{Ti} / \mathrm{Fe}] \mathrm{II} . . . . . . . . . .$. & 12 & 0.15 & 0.06 & 0.05 & EWs \\
\hline$[\mathrm{Cr} / \mathrm{Fe}] \mathrm{I} \ldots \ldots \ldots \ldots$ & 2 & -0.41 & 0.21 & 0.15 & EWs \\
\hline$[\mathrm{Mn} / \mathrm{Fe}] \mathrm{I} \ldots \ldots \ldots \ldots$ & 2 & -0.26 & 0.10 & 0.06 & EWs \\
\hline$[\mathrm{Sr} / \mathrm{Fe}] \mathrm{II} \ldots \ldots \ldots \ldots$ & 2 & 0.34 & 0.24 & 0.24 & EWs \\
\hline$[\mathrm{Y} / \mathrm{Fe}] \mathrm{II} \ldots \ldots \ldots \ldots . . .$. & 1 & $<0.91$ & $\ldots$ & $\ldots$ & EWs \\
\hline$[\mathrm{Zr} / \mathrm{Fe}] \mathrm{II} . . . \ldots \ldots \ldots$ & 1 & $<1.22$ & $\ldots$ & $\ldots$ & EWs \\
\hline$[\mathrm{Ba} / \mathrm{Fe}] \mathrm{II} . . . . . . . . .$. & 3 & 1.46 & 0.20 & 0.20 & EWs \\
\hline$[\mathrm{La} / \mathrm{Fe}] \mathrm{I} \ldots \ldots \ldots \ldots$ & & 1.8 & $\ldots$ & 0.2 & Syn \\
\hline$[\mathrm{Eu} / \mathrm{Fe}] \mathrm{I} \ldots \ldots \ldots \ldots$ & & $<1.1$ & $\ldots$ & 0.1 & Syn \\
\hline$[\mathrm{Pb} / \mathrm{Fe}] \mathrm{I} \ldots \ldots \ldots \ldots$ & & 3.3 & $\ldots$ & 0.1 & Syn \\
\hline${ }^{12} \mathrm{C} /{ }^{13} \mathrm{C} \ldots \ldots \ldots \ldots$. & & 6 & $\ldots$ & 1 & Syn \\
\hline $\mathrm{C} / \mathrm{O} \ldots \ldots \ldots \ldots \ldots$ & & 100 & $\cdots$ & $\ldots$ & Syn \\
\hline $\mathrm{N} / \mathrm{O} \ldots \ldots \ldots \ldots \ldots$ & & 8 & $\ldots$ & $\ldots$ & Syn \\
\hline
\end{tabular}

a Number of lines over which the abundance was averaged.

b Includes non-LTE corrections according to the prescriptions of Gratton et al. 1999.

stronger than in turnoff stars, because of the combination of a stronger ionizing flux and low surface gravity, in particular RR Lyrae stars. More recently, analysis of even hotter stars on the blue horizontal branch of several globular clusters (at temperatures of about $8000 \mathrm{~K}$ ) by Behr (1999) also confirms that a good equilibrium of ionization for Fe can be obtained assuming LTE (and atmospheric parameters derived from the location of the star in the color-magnitude diagram). According to Gratton et al., non-LTE corrections for such stars are expected to be a factor of at least 5 larger than in turnoff stars. We conclude that corrections for departures from LTE should be small for Fe, and we will neglect them hereafter.

The Al abundance has been corrected to account for nonLTE effects by using values interpolated from Table 1 of Baumüller \& Gehren (1997). Hyperfine structure was taken into account for Mn (data from Booth, Shallis, \& Wells 1983) and Ba, for which we used data from Steffen (1985).

\subsection{Spectral Synthesis}

Abundances for $\mathrm{Li}, \mathrm{C}, \mathrm{N}, \mathrm{Eu}, \mathrm{La}$, and $\mathrm{Pb}$ were derived from spectral synthesis, because features due to these elements are either very weak or somewhat blended with nearby lines due to other species. As a general rule, line lists used in these spectral syntheses were derived as follows. We extracted from Kurucz's database (Kurucz 1995b) lines of neutral and singly ionized atomic species for which the potential of the upper level was smaller than the ionization potential. We also included the molecular lines of $\mathrm{CN}$ and of the hydrides $\mathrm{CH}, \mathrm{NH}$, and $\mathrm{OH}$. For the hydrides we considered only lines with excitation potential E.P. $\leq 1.5 \mathrm{eV}$. In fact, since these molecules are easily dissociated, the upper levels for transitions starting at higher excitational levels have energies that are much higher than the dissociation energy and are undetectable in both the solar spectrum and in our observed spectra. We supplemented these lists with a few additional lines that are listed in the solar tables (Moore, Minnaert, \& Houtgast 1966) but are missing from the Kurucz database. Most of these lines are unidentified features; we arbitrarily assumed them to be Fe I lines with E.P. $=3.5 \mathrm{eV}$. The $\log g f$ values of the atomic transitions from which we measured the abundances were taken from the atomic physics literature (see the following sections for details and references) and left untouched, while the transition probabilities of the neighboring lines were adjusted by matching the solar spectrum. A synthetic spectrum of the wavelength range of interest was calculated using a Kurucz model for the solar atmospheric parameters. The $\log g f$ values were adjusted to reproduce the observed solar spectrum, taken from Kurucz's (Kurucz, Furenlid, \& Brault 1984) solar flux atlas. In some cases small offsets (of the order of $5 \times 10^{-3} \AA$ ) in the wavelengths were also applied. With the exception of $\mathrm{CH}$ and $\mathrm{CN}$, whose cases are discussed in $\S \S 4.3 .1-\oint \S 4.3 .3$, molecules were treated as contaminants. To reproduce the broadening mechanisms (instrumental, macroturbulence, and rotation), the calculated synthetic spectra were convolved with a Gaussian. The FWHM for the Gaussian was chosen according to the observed spectra. Once acceptable agreement with the solar spectra was reached, the line list was used to calculate the synthetic spectrum for the program star and thus to measure the abundance of the element of interest.

The observed data for HE 0024-2523 were smoothed by convolving the raw spectra with a Gaussian of FWHM = $0.1 \mathrm{~A}$. Whenever possible, the observed spectrum that was compared with the synthesis was the sum of the HIRES 2000 September and UVES 2001 August data. Whenever meaningful and possible, we also compared the spectrum of HE 0024-2523 with that of HE 2344-2800, taken in 2000 September, whose analysis was performed in Paper II. The two stars have very similar atmospheric parameters (HE 0024-2523: 6625/4.3/-2.70/1.40; HE 2344-2800: 6625/ $4.3 /-2.56 / 1.42$ ), so that the differences between the two spectra can be attributed almost exclusively to the chemical peculiarity of HE 0024-2523. In the text below we note when, because of the differences in the wavelength coverage, only the UVES spectrum was used.

\subsubsection{Abundance}

To measure the carbon abundance of HE 0024-2523, we calculated a synthetic spectrum from 4300 to $4340 \AA$. This region includes the band head of the (0-0), (1-1), (2-2) bands of the $\mathrm{A}^{2} \Delta-\mathrm{X}^{2} \Pi$ transitions of $\mathrm{CH}$. The dissociation potential used was $3.465 \mathrm{eV}$ from Brzozowski et al. (1976), and the $\log g f$ values of the electronic transitions were modified to reproduce the solar spectrum by using the solar abundance of carbon from Anders \& Grevesse (1989) and the Kurucz solar spectrum. We found a corrective factor of -0.3 dex in $\log g f$ and a shift of $-0.05 \AA$ in wavelength.

We then computed the synthetic spectra with atmospheric parameters appropriate for $\mathrm{HE} \mathrm{0024-2523}$ and carbon abundances from $[\mathrm{C} / \mathrm{Fe}]=+2.0$ to $[\mathrm{C} / \mathrm{Fe}]=+3.0$ in inter- 


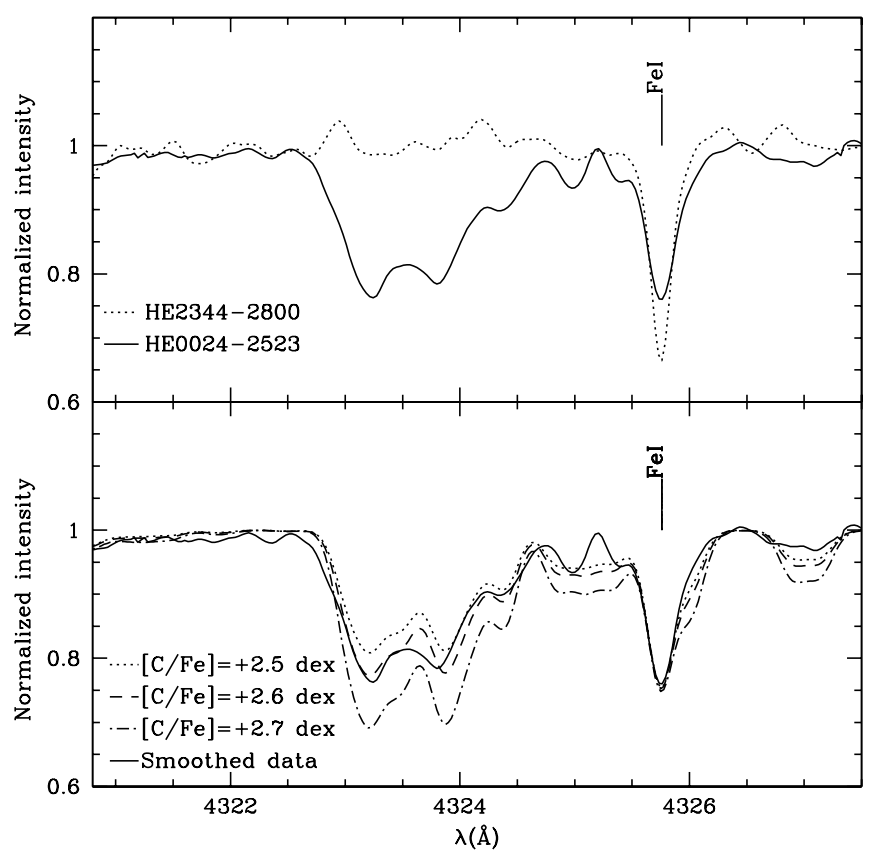

FIG. 5.-Comparison between observed and synthetized spectra for a spectral region within the $G$ band. The continuous line is the observed spectrum. Top: Comparison of the spectra of the program star (continuous line) and HE 2344-2800 (dashed line), which have almost identical atmospheric parameters. The $Q$ band head, which is undetectable in the normal star, is extremely strong in the program star. Note the difference in the shape of the $\mathrm{Fe}$ I line at $4325.78 \mathrm{~A}$, due to the nonnegligible rotational velocity of the program star $\left(\sim 9.7 \mathrm{~km} \mathrm{~s}^{-1}\right)$. Bottom: Comparison of the observed spectrum for HE 0024-2523 with three synthetic spectra computed with $[\mathrm{C} / \mathrm{Fe}]=+2.5,+2.6$, and $+2.7 \mathrm{dex}$.

vals of 0.1 dex. Figure 5 (bottom) shows the comparison between the observed spectrum and the synthetic ones. For the sake of clarity we have shown the synthetic spectra only for the cases $[\mathrm{C} / \mathrm{Fe}]=+2.5,+2.6$, and +2.7 . The overall best fit was achieved for $[\mathrm{C} / \mathrm{Fe}]=+2.6 \pm 0.1$. It is worth noting that the plotted synthetic spectra are the result of three separate steps. We first measured the carbon abundance using the solar isotopic ratio ${ }^{12} \mathrm{C} /{ }^{13} \mathrm{C}$; then, assuming this ${ }^{12} \mathrm{C}$ abundance, we measured the isotopic ratio as described in the following section, and finally we recomputed the synthetic spectra to determine the carbon abundance using the measured isotopic ratio. The spectra with the correct isotopic ratio exhibit a better fit to the data over the entire range. Figure 5 (top) shows the comparison between the spectrum of HE 0024-2523 and the carbon-normal star HE 2344-2800, where $\mathrm{CH}$ lines are undetectable.

\subsubsection{Isotopic Ratios}

The wavelength ranges of the spectrum used for the measurement of the isotopic ${ }^{12} \mathrm{C} /{ }^{13} \mathrm{C}$ ratio are 4229 to $4239 \AA$ and 4362 to $4372 \AA$. The line list was prepared as described in the previous section for the line list associated with the $G$ band. We computed synthetic spectra for isotopic ratios ${ }^{12} \mathrm{C} /{ }^{13} \mathrm{C}$ from 3 to 10 . The best fit for the region 4229 to $4239 \AA$ was obtained for a value of ${ }^{12} \mathrm{C} /{ }^{13} \mathrm{C}=6 \pm 1$, while in the region 4362 to $4372 \AA$ we obtained ${ }^{12} \mathrm{C} /{ }^{13} \mathrm{C}=5 \pm 1$. We adopted the ratio ${ }^{12} \mathrm{C} /{ }^{13} \mathrm{C}=6 \pm 1$ because in the first spectral range the differences between the synthetic spectra due to the different assumed isotopic ratios are clearly visible (see Fig. 6). In the second spectral range the isotopic effects are more subtle, but the overall fit is just as good (see Fig. 7).

\subsection{3. $N$ Abundance}

The $\mathrm{N}$ abundance was derived from the spectral region 3881-3885 $\AA$, which includes the band head of the $\mathrm{CN}$ violet system. In this case the line list was extracted from Kurucz's database with the selection criteria and the optimization procedure described in $\S 4.3$. We used a dissociation potential of $7.66 \mathrm{eV}$ from Engleman \& Rouse (1975) and even in this case we found a corrective factor of -0.3 dex in the $\log g f$ values of the electronic transitions. We computed synthetic spectra using $[\mathrm{C} / \mathrm{Fe}]=+2.6$, and with $[\mathrm{N} / \mathrm{Fe}]$ abundances ranging from +1 to +2.5 in 0.1 dex increments. As shown in Figure 8, the best fit was obtained for $[\mathrm{N} / \mathrm{Fe}]=+2.1 \pm 0.1$.

\subsubsection{Ba and Eu Abundance: $r$ - or s-Process?}

In our analysis we found a strong Ba overabundance (for a comparison with the normal halo star, HE 2344-2800, see Fig. 9). To rule out the possibility that $\mathrm{Ba}$ is due to the $r$ process, we looked for other prominent features of $r$-process elements. The most suitable element for this test is $\mathrm{Eu}$ : its relative $r$-fraction in the solar system is about 93\% (Käppeler et al. 1990a, 1990b). The log $g f$ values for the Eu II line at $4129.72 \AA$ was taken from Lawler et al. 2001b. We also took the hyperfine and isotopic splitting constants used to prepare the line list from the same source and adopted a solar system isotopic ratio. As can be seen from Figure 10, there is no clear evidence of a line at $4129.72 \AA$. An upper limit to the $\mathrm{Eu}$ abundance of $[\mathrm{Eu} / \mathrm{Fe}] \leq+1.1 \pm 0.4$ is deduced.

While this limit is not known with great accuracy, an $r$ process origin of $\mathrm{Ba}$ can be excluded. In fact, the expected Eu abundance in the case of $r$-process enrichment would be $[\mathrm{Eu} / \mathrm{Ba}] \sim 0.8$, that is to say, for $[\mathrm{Ba} / \mathrm{Fe}]=+1.46$ we would expect $[\mathrm{Eu} / \mathrm{Fe}] \simeq+2.2$. This $\mathrm{Eu}$ abundance is not consistent with the observed data, as Figure 10 clearly shows. The dot-dashed line, which represents the synthetic spectrum for $[\mathrm{Eu} / \mathrm{Fe}]=+2.2$, is characterized by an Eu feature much stronger than that present in the spectrum of $\mathrm{HE}$ 0024-2523, even with a generous allowance for errors in the placement of the continuum.

\subsubsection{Pb Abundance}

Having ruled out the $r$-process origin of the $\mathrm{Ba}$ in $\mathrm{HE}$ 0024-2523, we expect the Ba to originate from the $s$-process. According to most nucleosynthesis models, the flow of the $s$-process at low metallicities, given the scarcity of seed nuclei, drains the $\mathrm{Zr}$ and $\mathrm{Ba}$ peaks and builds an excess of ${ }^{208} \mathrm{~Pb}$, which is at the end of the $s$-process path (Busso et al. 2001). We measured the $\mathrm{Pb}$ abundance from two of the strongest lines in our spectral range: 3683.4 and $4057.8 \AA$. The transition probabilities for the two lines were taken from Hauge \& Sørli 1973. ${ }^{15}$

15 These $\log g f$ values are those used by Anders \& Grevesse 1989, whose solar abundances are adopted in our analysis and synthesis. They find $\log \epsilon(\mathrm{Pb})=+1.85 \pm 0.05$. More recent values of $\log g f$ (Biémont et al. 2000 ) for those two lines are smaller by about $0.11 \mathrm{dex}$, and the solar abundance obtained is correspondingly higher, $\log \epsilon(\mathrm{Pb})=+2.00 \pm 0.06$. The two effects essentially cancel out, and given that we use the old $\log g f$ and old solar lead abundance, the $[\mathrm{Pb} / \mathrm{Fe}]$ abundance derived for the program star is not significantly affected. 


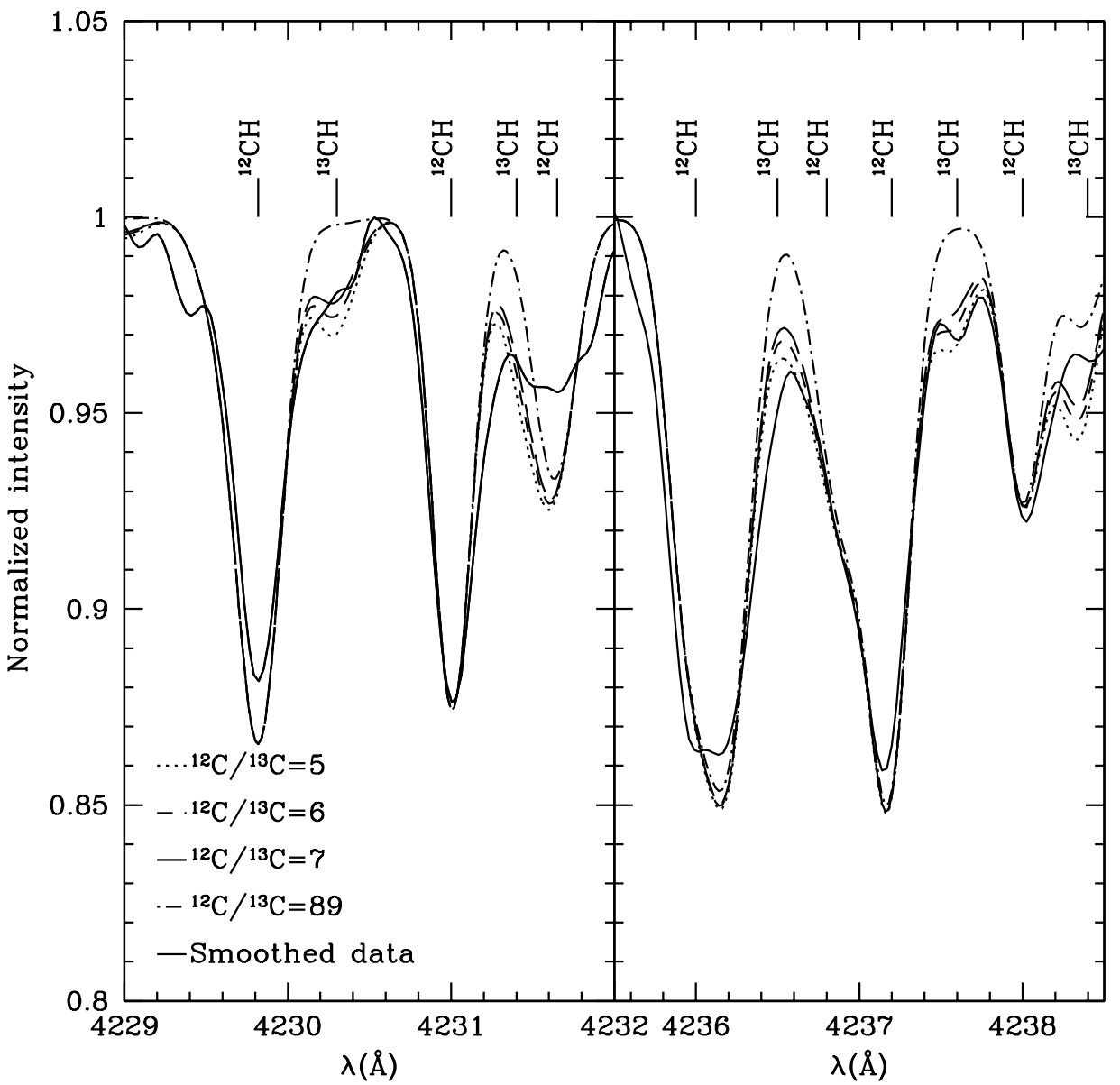

FIG. 6.-Comparison of observed and synthetized spectra in the regions 4229 to $4232 \AA$ and 4235.5 to $4239 \AA$. The solid line is the smoothed observed spectrum. The synthetized spectra are for ${ }^{12} \mathrm{C} /{ }^{13} \mathrm{C}=5,6$, and 7 ; a synthetic spectrum with solar isotopic $\mathrm{C}$ ratio ${ }^{12} \mathrm{C} /{ }^{13} \mathrm{C}=89$ is also plotted for comparison. Several ${ }^{12} \mathrm{CH}$ and ${ }^{13} \mathrm{CH}$ lines are shown to highlight the features on which the measurement is based.

Neither hyperfine structure nor isotopic splitting was included; the inclusion of these effects, which is probably worth doing when spectra of higher resolution and signalto-noise ratios are available, would tend to decrease the measured $\mathrm{Pb}$ abundance. However, the desaturation effect of the hyperfine splitting should not be very important in these lines, which are quite weak. Figure 11 shows the spectral region around the $3683.5 \AA$ line, together with three synthetic spectra calculated for $[\mathrm{Pb} / \mathrm{Fe}]=+3.2,+3.4$, and +3.6 , respectively. To illustrate just how strong the $\mathrm{Pb}$ line is, we have plotted a spectrum with a scaled solar lead content. The lead abundance measured from the $3683.4 \AA$ line is $[\mathrm{Pb} / \mathrm{Fe}]=+3.4 \pm 0.2$. No comparison with $\mathrm{HE}$ $2344-2800$ is shown as this line is outside the spectral range covered by our HIRES spectra.

The spectral region around the $4057.7 \AA$ line is covered by both UVES and HIRES spectra, so the signal-to-noise ratio is higher. Figure 12 (bottom) shows the observed spectra and the synthetic spectra computed for lead abundances $[\mathrm{Pb} / \mathrm{Fe}]=+3.1,+3.2$, and +3.3 , respectively, as well as the scaled solar value. From this line we measured a lead abundance of $[\mathrm{Pb} / \mathrm{Fe}]=+3.2 \pm 0.1$. The comparison with the spectrum of HE 2344-2800, in Figure 12 (top), shows a large feature in the spectrum of HE $0024-2523$ at $4057 \AA$ due to the lines of ${ }^{12} \mathrm{CH}, \mathrm{Fe}_{\mathrm{I}}$ and $\mathrm{Pb}$, while only the $\mathrm{Fe} \mathrm{I}$ line can be seen in the normal spectrum. Also, the blend of the two ${ }^{12} \mathrm{CH}$ lines at $\sim 4059.4 \mathrm{~A}$ is barely undetectable in that spectrum. We adopt the lead abundance for the program star from the mean of the two determinations: $[\mathrm{Pb} / \mathrm{Fe}]=+3.3 \pm 0.1$. This value might be slightly modified if the hyperfine and isotopic splitting were included. As mentioned, since the lines are quite weak, this would not lower the measured abundance by more than about 0.1 dex.

Thus, even taking the above factors into account, this is the most extreme $s$-process enrichment yet measured in an extremely metal-poor star. While the $\mathrm{Pb}$ abundance is extraordinarily high, the ratio $[\mathrm{Pb} / \mathrm{Ba}]=+1.84 \pm 0.22$, which represents the ratio of abundances between elements at the second and third s-process peaks, is the highest ever measured (see Table 7). The lead content in the Sun is about 1 $\mathrm{Pb}$ atom for every $3 \times 10^{5} \mathrm{Fe}$ atoms, while in this star it is about 1 in 100 . The previous record belonged to CS 31062-059 (Aoki et al. 2003), which has a metallicity $[\mathrm{Fe} / \mathrm{H}]=-2.38 \mathrm{dex}$, a lead content identical to that we have measured for $\mathrm{HE}$ 0024-2523 $([\mathrm{Pb} / \mathrm{Fe}]=+3.3$ $\pm 0.24)$, but a ratio $[\mathrm{Pb} / \mathrm{Ba}]=+1.19 \pm 0.14$.

\subsubsection{La Abundance}

We measured the lanthanum abundance from the 3988.5 and $4333.8 \AA$ lines of La II. The $\log g f$ values and the hyperfine splitting constant for these lines were taken from Lawler, Bonvallet, \& Sneden 2001a. We did not include isotopic shifts, since in the Sun the isotope ${ }^{159} \mathrm{La}$ accounts for 


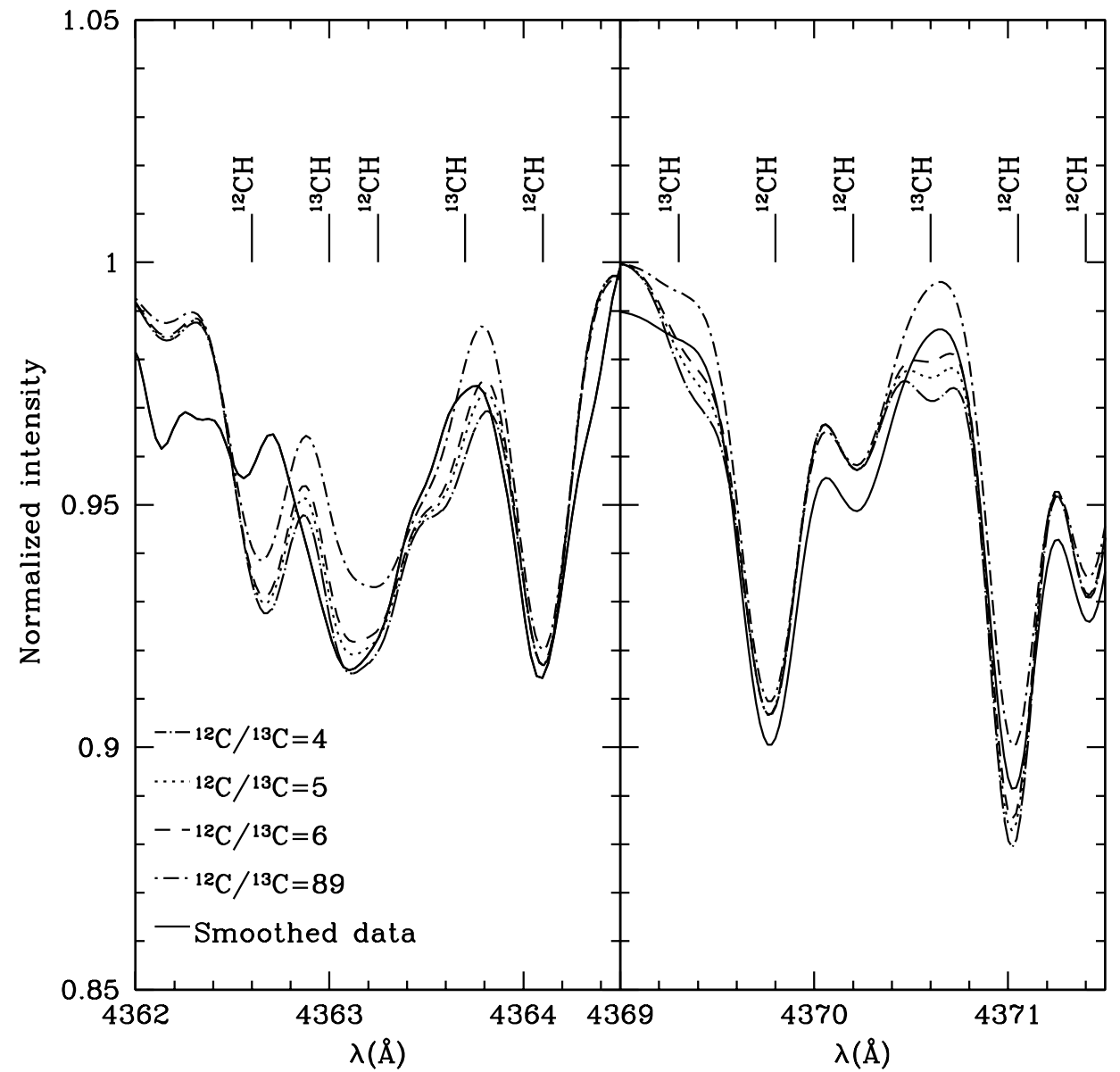

FIG. 7.-Same as Fig. 6 but for different spectral ranges, (4362 to $4364.5 \AA$ and 4369 to $4371.5 \AA$ ). The continuous line is the smoothed observed spectrum. The synthetized spectra are for ${ }^{12} \mathrm{C} /{ }^{13} \mathrm{C}=4,5$, and 6 ; a spectrum with solar isotopic $\mathrm{C}$ ratio ${ }^{12} \mathrm{C} /{ }^{13} \mathrm{C}=89$ is also plotted for comparison.

more than $99.9 \%$ of the total La content. The abundance measured from both lines is $[\mathrm{La} / \mathrm{Fe}]=+1.8 \pm 0.2$ (see Figs. 13 and 14). The error is quite large because the signal-tonoise ratio in the spectral region around both lines is not very good.

\subsubsection{Li Abundance}

We measured the $\mathrm{Li}$ abundance of HE 0024-2523 using the $6707 \AA$ resonance line. We inspected this element not because it is expected to participate in $s$-process nucleosynthesis, but because of recent results reported by

TABLE 7

[Pв/Ba] Ratios and Identified Periods for Extremely Metal-poor $s$-Process Enriched Stars

\begin{tabular}{|c|c|c|c|c|c|c|c|c|c|}
\hline Star & $\begin{array}{c}{[\mathrm{Fe} / \mathrm{H}]} \\
(\mathrm{dex})\end{array}$ & $\begin{array}{c}\sigma \\
(\operatorname{dex})\end{array}$ & $\begin{array}{c}{[\mathrm{Pb} / \mathrm{Fe}]} \\
(\mathrm{dex})\end{array}$ & $\begin{array}{c}\sigma \\
(\operatorname{dex})\end{array}$ & $\begin{array}{c}{[\mathrm{Pb} / \mathrm{Ba}]} \\
(\mathrm{dex})\end{array}$ & $\begin{array}{c}\sigma \\
(\operatorname{dex})\end{array}$ & ${ }^{12} \mathrm{C} /{ }^{13} \mathrm{C}$ & $v_{\mathrm{rad}}{ }^{\mathrm{a}}$ & $\begin{array}{l}\text { Period } \\
\text { (days) }\end{array}$ \\
\hline CS $29526-110^{\mathrm{b}}$. & -2.38 & 0.16 & 3.30 & 0.24 & 1.19 & 0.29 & & Yes & \\
\hline CS $22898-027^{\mathrm{b}}$ & -2.26 & 0.11 & 2.84 & 0.19 & 0.61 & 0.24 & $>20:^{c}$ & No & \\
\hline CS 31062-012 & -2.55 & 0.11 & 2.40 & 0.19 & 0.38 & 0.25 & & & \\
\hline 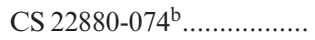 & -1.93 & 0.11 & 1.90 & 0.19 & 0.59 & 0.25 & $>40^{\mathrm{c}}$ & No & \\
\hline CS $31062-050^{\mathrm{b}} \ldots \ldots \ldots \ldots \ldots \ldots$ & -2.31 & 0.14 & 2.90 & 0.24 & 0.60 & 0.28 & & & \\
\hline CS 22942-019 b.. & -2.64 & 0.14 & $<1.60$ & $\ldots$ & $<-0.32$ & $\ldots$ & $300^{\mathrm{c}}$ & Yes & $2800^{\mathrm{c}}$ \\
\hline HD $196944^{b} \ldots$ & -2.25 & 0.19 & 1.90 & 0.24 & 0.80 & 0.31 & & & \\
\hline CS 30301-015 & -2.64 & 0.18 & 1.70 & 0.24 & 0.25 & 0.29 & & & \\
\hline HE $0024-2523^{\mathrm{d}} \ldots \ldots \ldots \ldots . . . .$. & -2.72 & 0.12 & 3.30 & 0.10 & 1.84 & 0.22 & 6 & Yes & 3.14 \\
\hline LP $625-44^{\mathrm{e}}$ & -2.72 & 0.16 & 2.60 & 0.22 & -0.21 & 0.33 & & Yes & \\
\hline 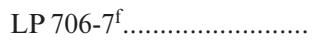 & -2.74 & 0.16 & 2.28 & 0.20 & 0.27 & 0.21 & & No & \\
\hline
\end{tabular}

a Indicates whether radial velocity variation was detected.

b Aoki et al. 2003.

c Preston \& Sneden 2001.

d Present work.

e Aoki et al. 2002a.

f Aoki et al. 2001. 


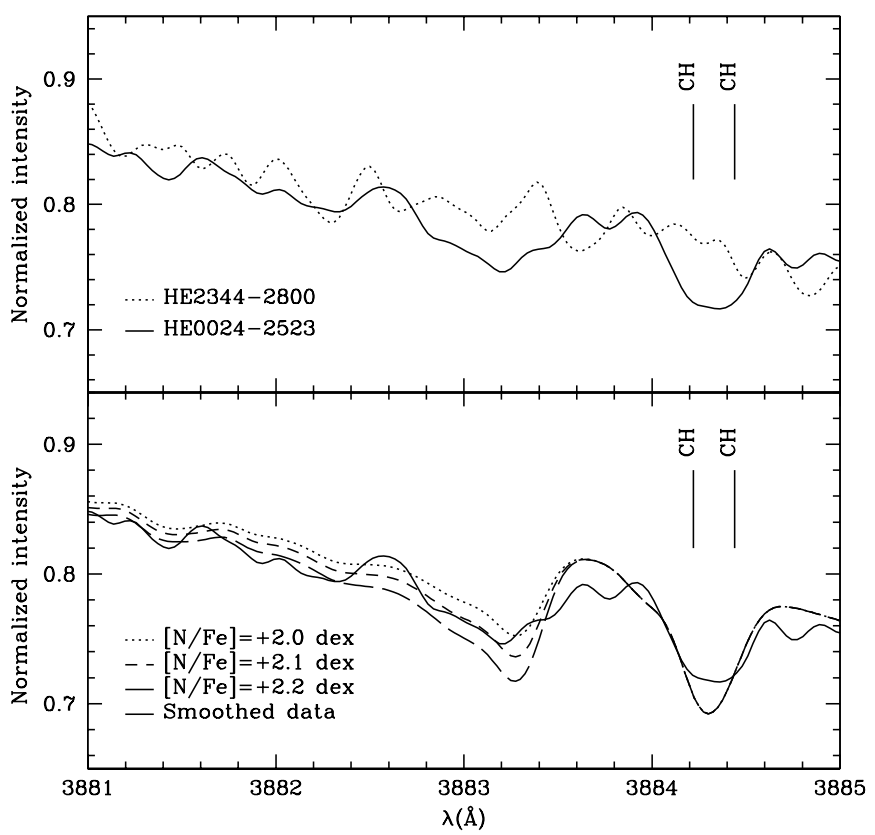

FIG. 8.-Bottom: Comparison of observed and synthetized spectra in the region of the UV CN band head. The solid line represents the smoothed observed spectrum. The synthetic spectra plotted are those for $[\mathrm{N} / \mathrm{Fe}]=+2.0,+2.1$, and +2.2 dex. The continuum is heavily affected by the wings of the Balmer line H8 at $3889.9 \AA$ A. Top: Program star spectrum in the same spectral range, together with the spectrum of our comparison star, HE 2344-2800, which does not show evidence of CN band features. Note also the $\mathrm{CH}$ feature at $\sim 3884.3 \AA$ is completely lacking in the spectrum of HE 2344-2800.

Ryan et al. (2002). These authors, on the basis of the analysis of a sample of 18 halo main-sequence turnoff stars, suggest that the $\mathrm{Li}$ abundance in their sample seems to be anticorrelated with rotational velocity. Figure

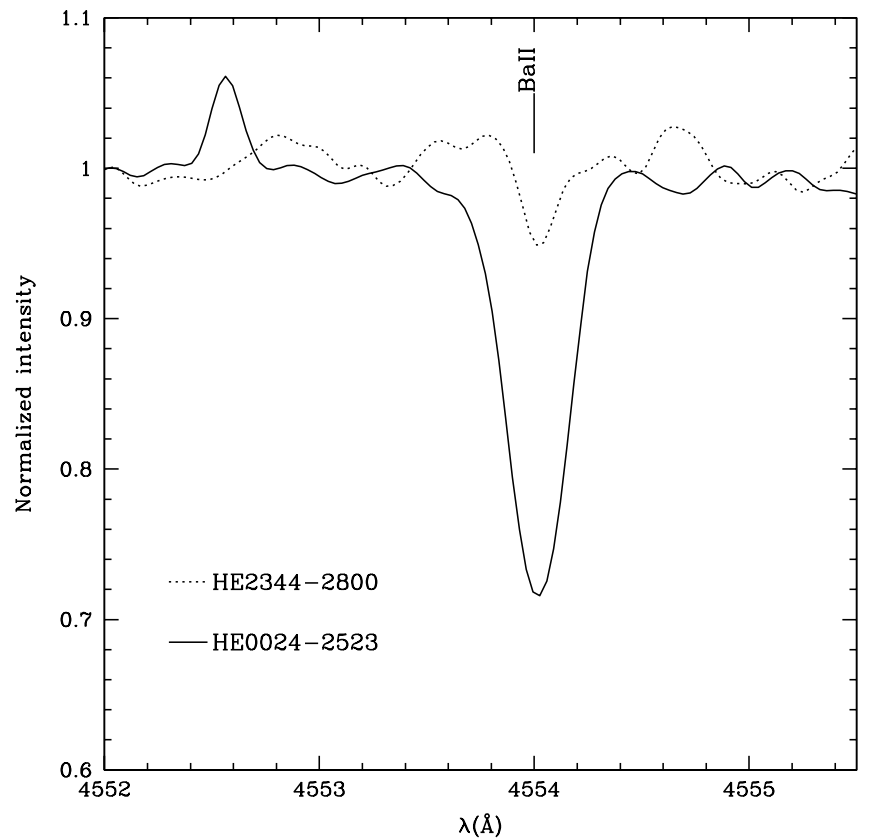

FIG. 9.-Comparison between the smoothed spectra of HE 0024-2523 and HE 2344-2800 in the spectral region around the Ba II $4554 \AA$ line. The difference is remarkable and reflects the measured $\mathrm{Ba}$ abundances: $[\mathrm{Ba} / \mathrm{Fe}] \mathrm{II}=+1.46 \pm 0.2 \mathrm{dex}$ in HE $0024-2523$ (see $\S 4.3$ for details) and $[\mathrm{Ba} / \mathrm{Fe}] \mathrm{II}=-0.55 \pm 0.2 \mathrm{dex}$ for HE 2344-2800.

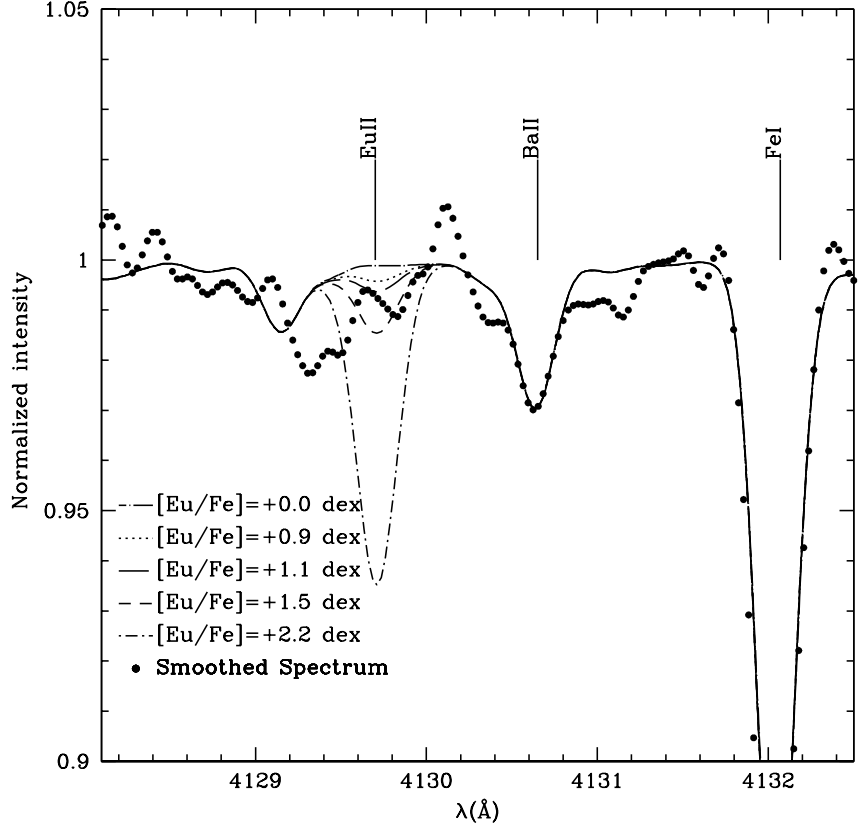

FIG. 10.-Comparison of the observed spectrum with synthetized ones for the Eu II line at $4129.72 \AA$. The dots represent the smoothed observed spectrum. The synthetic spectra were computed for $[\mathrm{Eu} / \mathrm{Fe}]=+0.9,+1.1$, and +1.5 dex. Also shown is a spectrum calculated with solar $[\mathrm{Eu} / \mathrm{Fe}]$ and with $[\mathrm{Eu} / \mathrm{Fe}]=+2.2$ dex which, given the measured Ba abundance of $[\mathrm{Ba} / \mathrm{Fe}]=+1.46 \mathrm{dex}$, represents the expected $\mathrm{Eu}$ abundance in case of $r$-process elements enrichment.

15 displays the observed and synthetized spectra in the region of the Li doublet; no definite feature was detected at $6707 \AA$, hence we deduce an upper limit of $\log \epsilon(\mathrm{Li})=+1.4$.

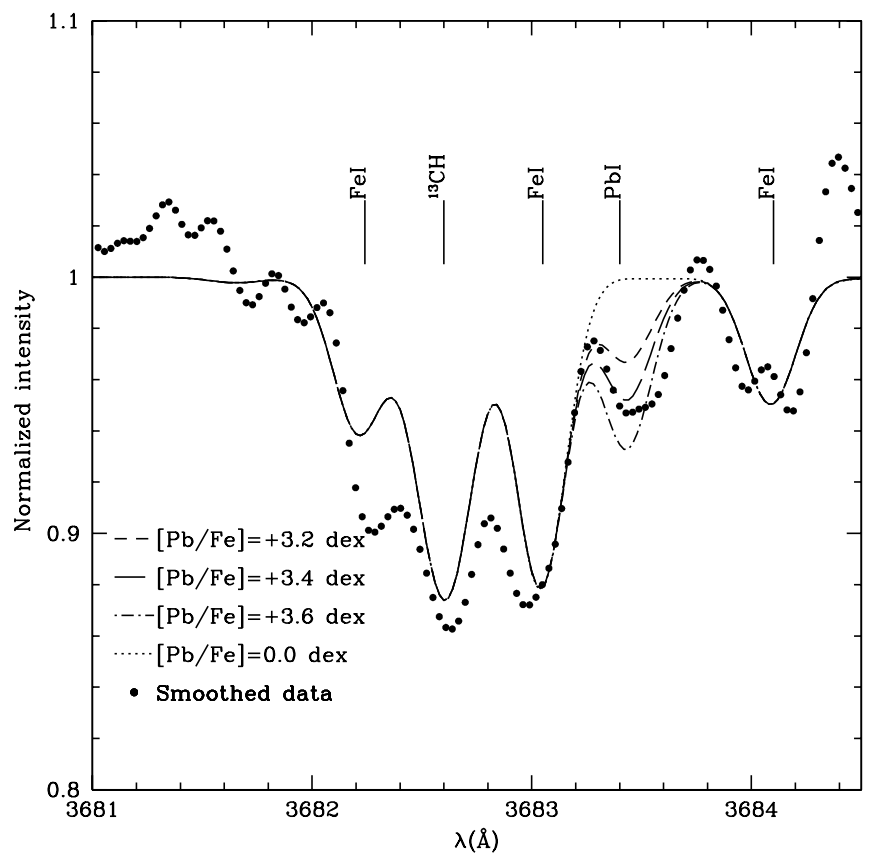

FIG. 11.-Comparison of the observed spectrum with the synthetic spectra in the spectral region around the $\mathrm{Pb}$ line at $3683 \AA$. The smoothed observed spectrum is represented by the dots. Synthetic spectra plotted are for lead scaled solar abundance, $[\mathrm{Pb} / \mathrm{Fe}]=+3.2,+3.4$, and +3.6 dex. The spectrum appears very noisy because it is at the blue end of the region observed and only data from UVES were available, as this line is outside the wavelength range covered by our HIRES spectra. 


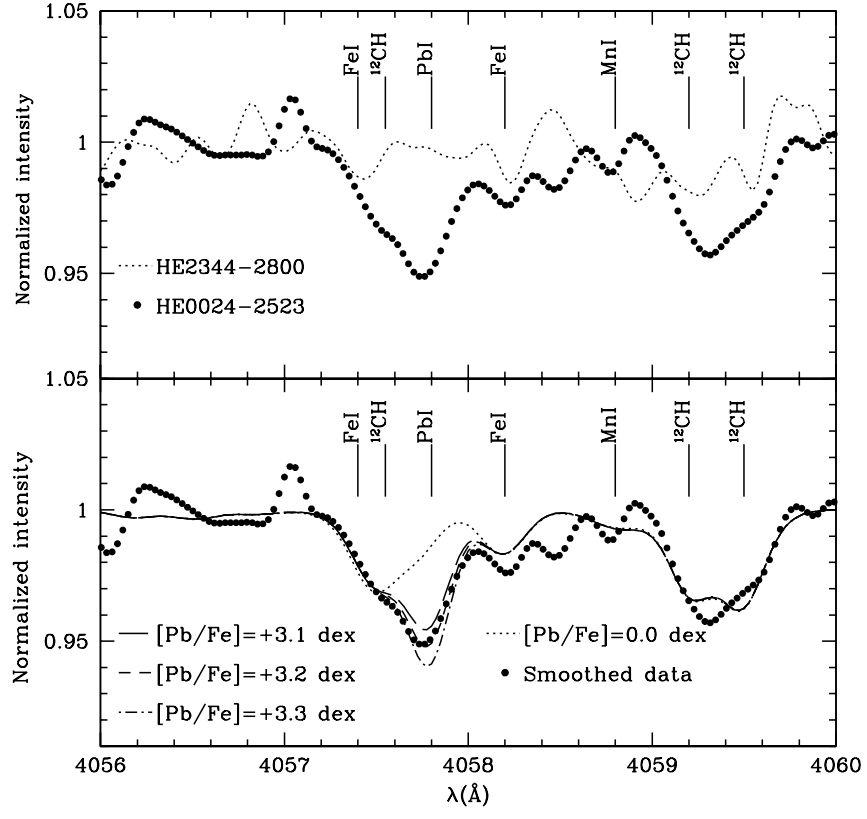

FIG. 12.-Bottom: Comparison of the smoothed observed spectrum with the synthetic ones in the region around the $\mathrm{Pb}$ line at $4057 \AA$. Synthetic spectra plotted are for lead scaled solar abundance, $[\mathrm{Pb} / \mathrm{Fe}]=+3.1,+3.2$, and +3.3 dex. Top: Comparison with the spectrum of the normal abundance pattern star HE 2344-2800. Note the difference in the Pb I $4057.7 \AA$ line and in the $\mathrm{CH}$ features.

\subsection{Abundance Summary}

Table 6 shows a summary of measured elemental abundances for HE 0024-2523. Column (6) indicates whether the listed abundance was derived via a line-by-line analysis

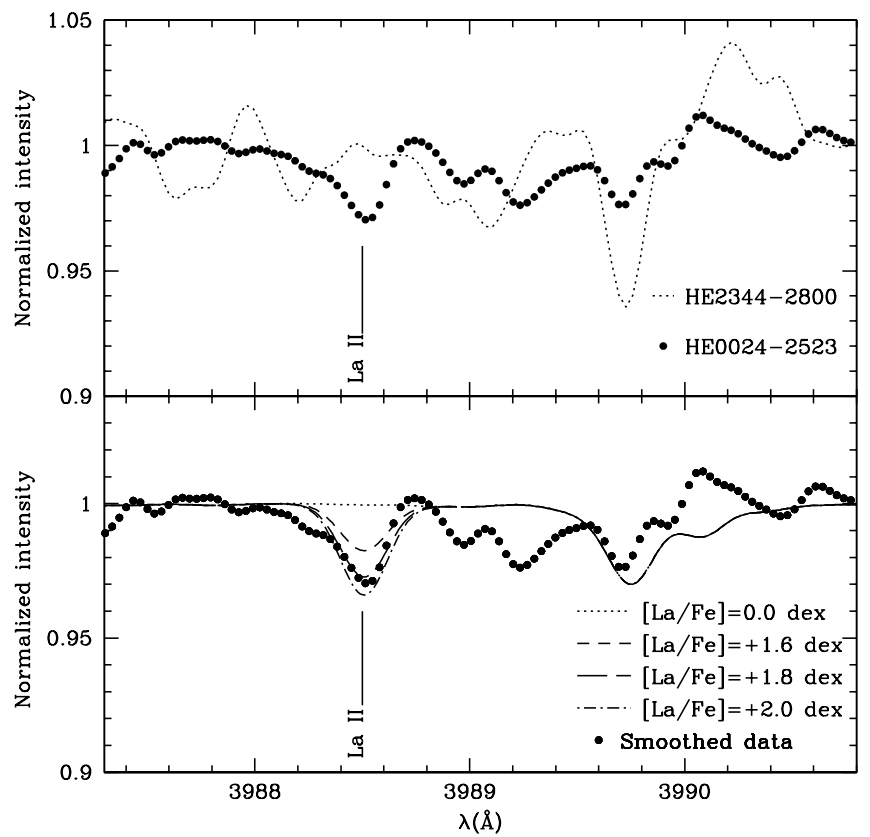

FIG. 13.- Spectral region around the La II line at 3988.5 A. Bottom: Comparison of the smoothed spectrum of the program star with synthetic ones computed for $\mathrm{La}$ abundances of $[\mathrm{La} / \mathrm{Fe}]=+1.6,+1.8$, and $+2.0 \mathrm{dex}$ and a synthetic spectrum with scaled solar La abundance. The signal-tonoise ratio in this spectral region is not very high. Top: Comparison of the spectrum of the program star with that of the template star HE 2344-2800, where the La feature is not detected.

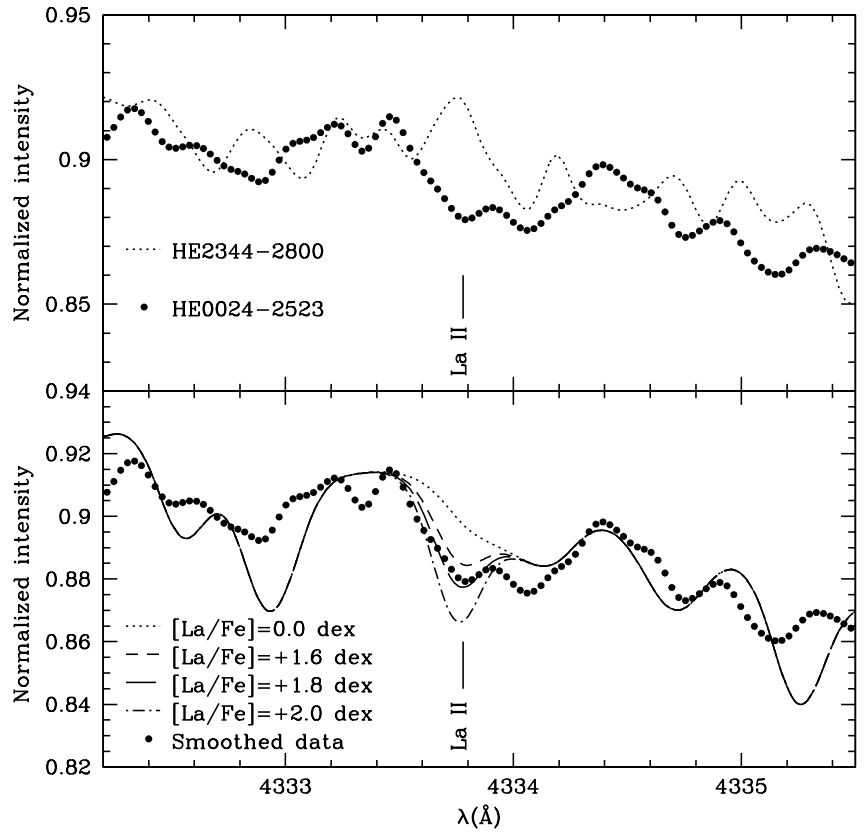

FIG. 14. - Same as Fig. 13 but for the spectral region around the La II line at $4333.8 \AA$. The spectrum is quite noisy, and the continuum is heavily affected by the wings of the Balmer line $\mathrm{H} \gamma$, which is well reproduced by our synthetic spectra.

of measured equivalent widths (indicated by "EWs") or with spectral synthesis ("syn"). For the abundance obtained from the analysis of equivalent widths, the rms is the dispersion of abundances as measured from the different lines, while the listed errors take into account the scatter in the abundances derived from the lines (i.e., the rms divided by the square root of the number of the lines) and the effect

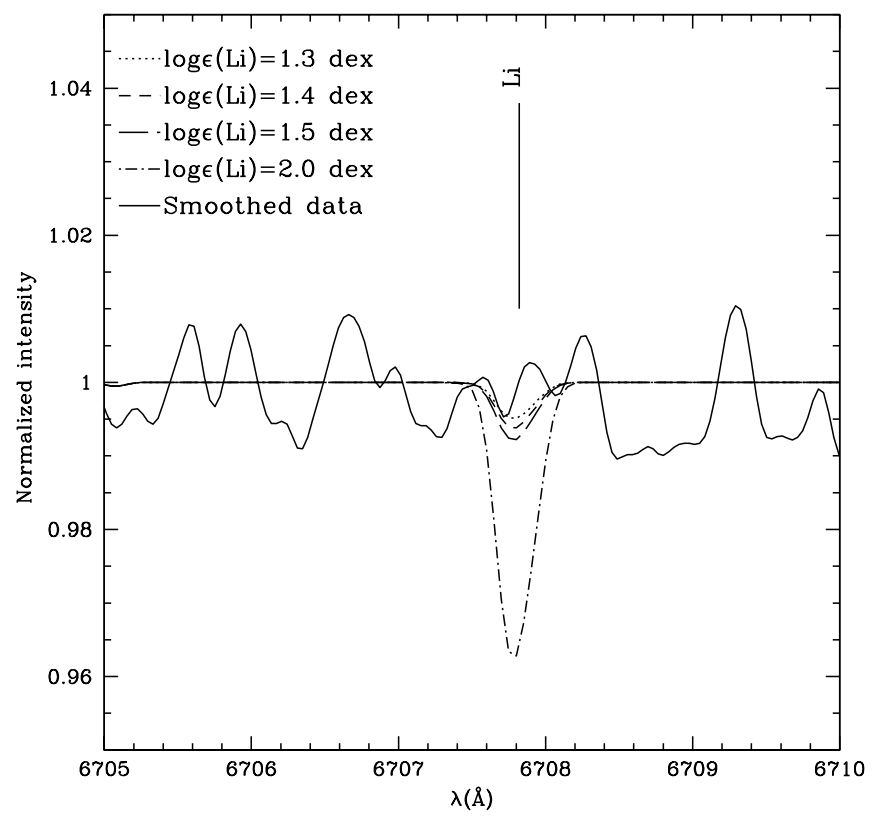

FIG. 15.-Comparison between the observed data and the synthetic spectra for the $\mathrm{Li}$ resonance line at $6707 \AA$. Note the scale is extremely blown up. The synthetized spectra are for $\log \epsilon(\mathrm{Li})=+1.3,+1.4,+1.5$, and +2.0 . The latter roughly represents the primordial $\mathrm{Li}$ abundance. The difference between the expected intensity of the primordial Li feature and that observed suggests that some Li depletion must have taken place. 
of the uncertainties in the atmospheric parameters, as explained in $\S 4.3$. Also reported are the $\mathrm{C}$ isotopic ratio and the ratios $\mathrm{C} / \mathrm{O}$ and $\mathrm{N} / \mathrm{O}$, by number.

\section{DISCUSSION}

Our results clearly indicate that the chemical composition of the atmosphere of HE 0024-2523 is the outcome of an $s$ process enrichment. The abundance pattern observed is very extreme, especially as far as $\mathrm{Pb}$ is concerned. Canonical $s$-process nucleosynthesis is associated with the AGB stage of evolution, during which the star is powered by hydrogen and helium burning in two shells located above a degenerate core (see, e.g., Iben \& Renzini 1983). Late on the AGB, intermediate-mass stars undergo recurrent thermal instabilities of the He shell, in which partial He burning occurs convectively over short periods of time, sweeping across the whole region that lies between the $\mathrm{H}$ and the He shells. During this phase, the material from the $\mathrm{He}$ intershell that is enriched in $\mathrm{C}$ and $s$-process elements is taken to the surface by the extension of the envelope convection (third dredgeup). Even later in the evolution, as the result of stellar winds, which progressively strip its envelope, only the degenerate core is left and the star will become a white dwarf (see Busso et al. 2001 for a thorough discussion of $s$-process elements nucleosynthesis).

As discussed in Paper I, the observational data indicate that HE 0024-2523 is presently going through its upper main-sequence phase and thus is not capable of having produced the abundance pattern we have measured. The necessity of an external origin of some of the detected elements and the clear binarity of the object suggests that HE 0024-2523 is an extrinsic CH star, i.e., the atmosphere of the star observed has been polluted by material that has been processed by a more massive companion that already underwent its AGB phase and is now a white dwarf (and undetectable in our present observations). This model is further supported by consideration of the orbital solution, as discussed in $\S 3.3$ above.

\subsection{Orbital Parameters}

It is noteworthy that the orbital period of HE 0024-2523 is unusually short, compared with those typical of this class of object, which is several hundred days (McClure \& Woodsworth 1990). This anomaly suggests that HE 0024-2523 went through a common envelope phase at an earlier stage of the binary interaction, when the more massive component expands so rapidly that mass transfer is unstable, and the companion is engulfed in the atmosphere of the donor star. The ejection of the envelope requires energy to be removed from the orbit, and the latter shrinks, shortening its period (see, e.g., Sandquist et al. 1998).

This picture could explain the peculiarity of this star as the result of a more pronounced difference between the original masses of the components of the binary, since the common envelope phase is the consequence of unstable mass transfer. This is consistent with the measured $\mathrm{C}$ isotopic ratio, which, as discussed later in this section, suggests an initial mass for the evolved companion of at least $\sim 3 M_{\odot}$. Had the stars been more similar in mass, the mass transfer might have taken place quiescently, and the orbit would have shrunk much less, probably reaching the typical $\mathrm{CH}$ star period.

\subsection{Testing Theoretical Models}

We now compare our measured abundances for the $s$ process elements with those predicted from an evolution model computed for a metallicity of $[\mathrm{Fe} / \mathrm{H}]=-2.6$ and mass $M=1.5 M_{\odot}$ (R. Gallino 2002, private communication), which we consider here representative of the now evolved companion of the observed star. To compare the predictions with the observations, we assumed a dilution factor, the percentage of atmospheric material that has been accreted by the observed star from the donor star, of $10 \%$. This assumption, while reasonable, is completely arbitrary and was set to match the observed carbon abundance. We also made the natural assumption that the original (i.e., preaccretion) abundance pattern of the observed star resembled that of a typical star of similar metallicity. For this purpose, we used the mean of the abundances measured in several stars at $[\mathrm{Fe} / \mathrm{H}] \sim-2.7$ (see $\S 5.2$ of Paper II for more details on the sample).

The comparison is shown in Figure 16. Several elements shown have negligible contribution from the shell nucleosynthesis ( $\mathrm{Mg}$ through $\mathrm{Fe}$ ), thus the fit is not very meaningful for them. The prediction in the case of $\mathrm{Pb}$ and $\mathrm{Ba}$, produced exclusively by AGB nucleosynthesis, is excellent, with the model falling well within the error bars. On the other hand, Sr is not reproduced particularly well. However, it should be kept in mind that the contribution of the original (preaccretion) Sr content to the final one has to be considered as well; the measured $\mathrm{Sr}$ abundance in metal-poor
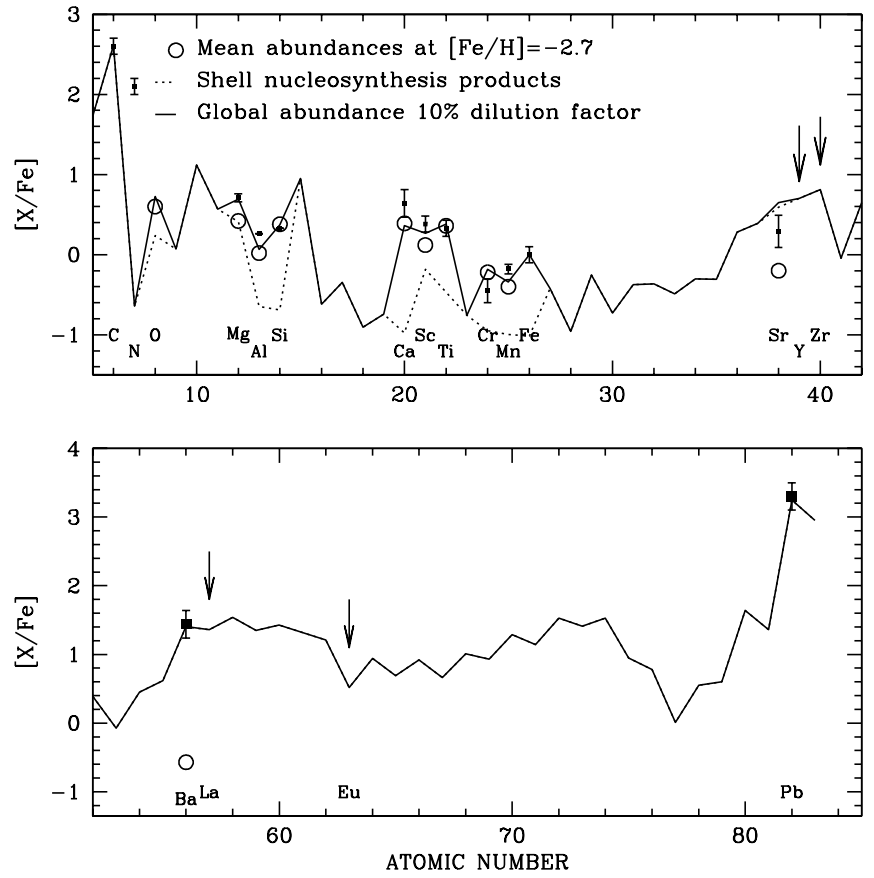

FIG. 16.-Comparison of our data with a nucleosynthesis model by R. Gallino (2000, private communication; for details of the model see Busso et al. 2001). The model was calculated for a metallicity of $[\mathrm{Fe} / \mathrm{H}]=-2.6$ dex and mass $M=1.5 M_{\odot}$. The dashed line represents the predicted shell nucleosynthesis model abundance. The solid line is the resulting abundance for the star's atmosphere after accretion of material from the companion, assuming a dilution factor of $10 \%$. The squares are our measured abundances, while the arrows indicate the upper limits. The assumed original abundances of the program star are indicated by the circles (determined from the mean observed abundances for stars at $[\mathrm{Fe} / \mathrm{H}]=-2.7 \mathrm{dex}$ ). The abundances have been scaled to $[\mathrm{Fe} / \mathrm{H}]=-2.7$ dex. 
stars is known to have a large scatter. Thus, it is possible that the average $\mathrm{Sr}$ content that we assumed as representing the original abundance of this element does not pertain. The measured upper limits for the abundances of $\mathrm{Eu}, \mathrm{La}, \mathrm{Y}$, and $\mathrm{Zr}$ are indicated by arrows. All of them are consistent with the model.

While the model seems to reproduce satisfactorily our measurements for $s$-process elements, it fails to explain the low ${ }^{12} \mathrm{C} /{ }^{13} \mathrm{C}$ ratio, predicting a value of several hundred (R. Gallino 2002, private communiction). The low measured ratio $\left({ }^{12} \mathrm{C} /{ }^{13} \mathrm{C}=6 \pm 1\right)$ is quite unusual for $s$-process enriched material. In fact, the primary source of neutrons is thought to be the ${ }^{13} \mathrm{C}(\alpha, n){ }^{16} \mathrm{O}$ reaction, hence ${ }^{13} \mathrm{C}$ is expected to be highly depleted. The value of the isotopic ratio is only marginally larger than that of $\mathrm{CN}$ cycle equilibrium value $\left({ }^{12} \mathrm{C} /{ }^{13} \mathrm{C} \sim 3\right)$ and seems to suggest a signature of hot bottom burning (HBB hereafter). Since the observed star is still on the main sequence, it has not gone through the first dredge-up and all the carbon observed, both ${ }^{13} \mathrm{C}$ and ${ }^{12} \mathrm{C}$, presumably comes from the former AGB donor star.

On the basis of the hypothesis that the donor star underwent HBB before mass transfer took place, we can place a reasonable lower limit to the original mass of the donor star, which is the minimum mass for which HBB is expected to occur. This is $\sim 3 M_{\odot}$ (P. Marigo 2002, private communication) for the metallicity under discussion. This value is lower than the usual one, $\sim 4.5 M_{\odot}$ (Marigo 1998), because, as metallicity declines, the mass at which the temperature necessary for igniting HBB is reached also decreases (see Siess, Livio, \& Lattanzio 2002 for an extreme case at zero metallicity). It should be noted that the initial mass for the shell nucleosynthesis model we used for comparison, $M=1.5$ $M_{\odot}$, is smaller than the lower limit placed when taking into account the low ${ }^{12} \mathrm{C} /{ }^{13} \mathrm{C}$. $\mathrm{HBB}$, which would lower the aforementioned ratio, possibly approaching that of our measurement, is not achieved in the model.

A recent paper by Ventura, D'Antona, \& Mazzitelli (2002) presents the yields from AGB stars with $2.5 \leq$ $M / M_{\odot} \leq 6$ and $-2.0 \leq[\mathrm{Fe} / \mathrm{H}] \leq-0.3$. Their results seem to agree with our findings for HE 0024-2523, both for the isotopic $\mathrm{C}$ ratio and $\mathrm{C} / \mathrm{O}$ ratio. Nevertheless, the $\mathrm{N}$ abundance and the N/O ratio are not reproduced in these models, which predict that the $\mathrm{N}$ abundance should be about an order of magnitude larger than that of C. As shown in Figure 16, unlike the data in the literature, the extreme value of $[\mathrm{Pb} / \mathrm{Ba}]$ for HE $0024-2523$ is very well explained by models with the standard mass of the ${ }^{13} \mathrm{C}$ pocket (Busso et al. 2001), which predict $[\mathrm{Pb} / \mathrm{Ba}] \sim 2$. On the other hand, the values found in the literature (see Table 7) are lower and to be matched by the models would require a reduction of the mass of the ${ }^{13} \mathrm{C}$ pocket by factors 6 to 24 (Aoki et al. 2003). Although the data for the ${ }^{12} \mathrm{C} /{ }^{13} \mathrm{C}$ ratio in other similar stars are still very limited, it is noteworthy that the value for HE 0024-2523 is the lowest of the sample, consistent with the suggestion that the donor star had an unusually high initial mass, as discussed above.

\subsection{Comparison with Literature Data for s-Process- Enriched Extremely Metal-Poor Stars}

Figure 17 displays the ratio $[\mathrm{Pb} / \mathrm{Ba}$ ] for $\mathrm{HE} 0024-2523$ and for data from the literature (Aoki et al. 2000, 2002a, 2003) as a function of $[\mathrm{Fe} / \mathrm{H}]$. Aoki et al. (2003) suggested that the available data might show evidence of a decreasing

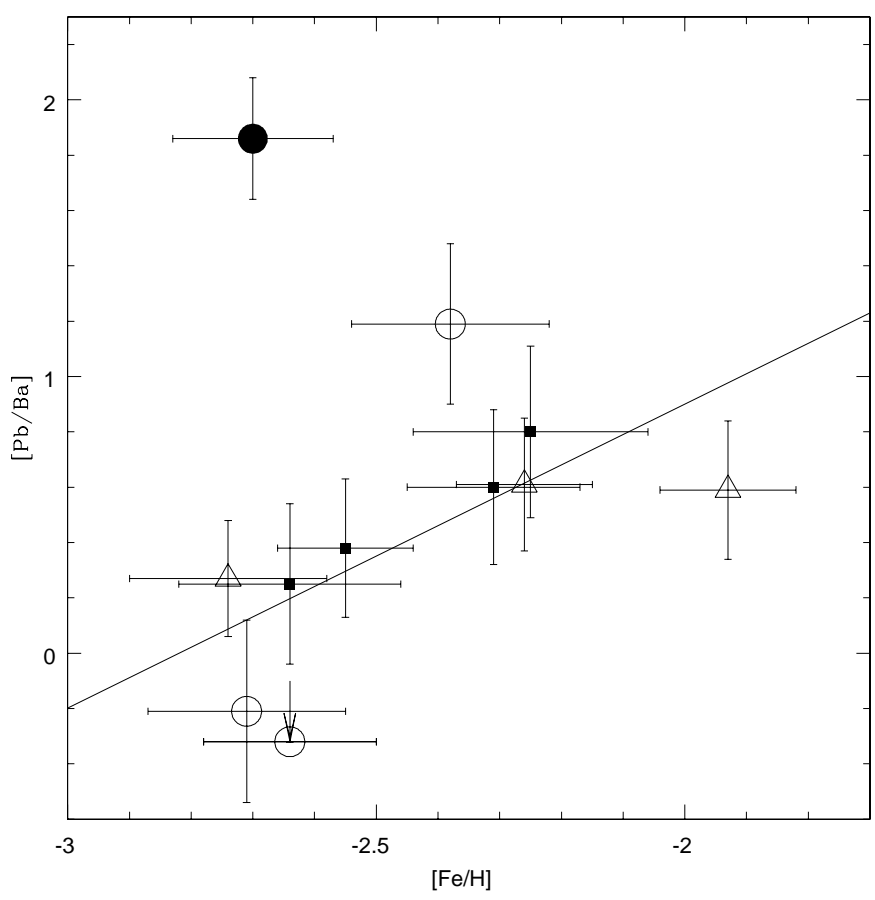

FIG. 17. - $[\mathrm{Pb} / \mathrm{Ba}]$ abundance as a function of $[\mathrm{Fe} / \mathrm{H}]$ for HE $0024-2523$ and literature data (Aoki et al. 2000, 2002a, 2003), showing HE 0024-2523 (filled circle), stars with detected radial velocity variation (circles), stars with no radial velocity variation over an interval of several years (open triangles; Preston \& Sneden 2001; Norris, Ryan, \& Beers 1997a) and stars with a single observation (squares). The addition of HE 0024-2523 to the sample seems to invalidate the correlation between $[\mathrm{Fe} / \mathrm{H}]$ and $[\mathrm{Pb} / \mathrm{Ba}]$ suggested by Aoki et al. (2003).

trend of $[\mathrm{Pb} / \mathrm{Ba}]$ with decreasing $[\mathrm{Fe} / \mathrm{H}]$. Our analysis seems not to support this hypothesis, at least not in all cases. It is quite clear from Figure 17 that, once the data point for HE $0024-2523$ is added, no correlation seems to be present. However, caution should be exerted when drawing any conclusion from this apparent lack of correlation. HE $0024-2523$ is the only star in the sample that likely went through a common envelope phase, which may well have influenced the nucleosynthesis history of the donor star. Thus, the exceptionally high $[\mathrm{Pb} / \mathrm{Ba}]$ for such a low metallicity $([\mathrm{Fe} / \mathrm{H}]=-2.7)$ with respect to those found in the literature could arise from the peculiar evolution of $\mathrm{HE}$ $0024-2523$ and not destroy the $[\mathrm{Pb} / \mathrm{Ba}]$ versus $[\mathrm{Fe} / \mathrm{H}]$ correlation suggested by Aoki et al. (2003). Presumably, the addition of more stars with measured $[\mathrm{Pb} / \mathrm{Ba}]$, especially at the lowest metallicity, will help clarify the situation.

Another noteworthy feature seen in Figure 17 is the larger scatter in $[\mathrm{Pb} / \mathrm{Ba}]$ about the regression line for stars with detected radial velocity variations. For these objects, the observed $s$-process enrichment is a signature of the nucleosynthesis that took place on the companions during their AGB phase and was then accreted by the observed stars. Thus, our results indicate that the ratio $[\mathrm{Pb} / \mathrm{Ba}]$ produced by the $s$-process in metal-poor stars $([\mathrm{Fe} / \mathrm{H}]<-1.9)$ shows a large scatter.

A possible explanation for this scatter could be a correlation between the $[\mathrm{Pb} / \mathrm{Ba}]$ ratio and the radial velocity variations. In fact, we could invoke the presence of a close companion as a factor affecting shell nucleosynthesis. If the orbital period is large, the donor star will undergo complete evolution along the AGB, and the composition of the 
accreting star will be dominated by material lost by the evolved star just before the final thermal pulse and planetary nebula ejection. On the other hand, a very short period, like the one observed in HE 0024-2523, indicates a past common envelope phase; during this stage, a large amount of mass is lost by the system, leading to a premature end of the thermal pulse phase of the donor. In this case the chemical composition of the accreting star will be dominated by material lost by the evolved star during earlier phases of the evolution along the AGB. This may have a big impact on $\mathrm{HBB}$ processes, affecting the abundances of ${ }^{12} \mathrm{C},{ }^{13} \mathrm{C}, \mathrm{N}$, and possibly even $\mathrm{O}$, and thus the entire $s$-process history, which relies on the ${ }^{13} \mathrm{C}$ pocket as a neutron source.

\subsection{HE 0024-2523: A Future Blue Straggler?}

A number of recent papers (e.g., Preston \& Sneden 2000; Carney et al. 2001; Ryan et al. 2001) considering a wide range of evidence (e.g., the observed high binary fraction among field blue stragglers, the distribution of observed orbital periods and eccentricities in metal-poor, carbonenhanced stars) have come to the conclusion that the formation of blue stragglers is closely connected to the presence (now or in the past) of a companion star that has undergone mass transfer. Ryan et al. (2001), in particular, have argued that $\mathrm{Li}$ depletion in mass transfer binaries (even those that are presently too cool to be referred to as blue stragglers) might account for the lack of $\mathrm{Li}$ observed in the field blue stragglers that they have either already evolved into or which they will eventually evolve into. Ryan et al. (2002) have recently provided clear evidence that a large fraction of halo main-sequence stars that exhibit extreme Li depletion also have substantial axial rotation, with $v \sin i$ in the range $5-8 \mathrm{~km} \mathrm{~s}^{-1}$. One is thus tempted to consider the observed properties of HE 0024-2523 and ask whether it might present an extreme example of these phenomena.

Clearly, the presence of carbon enhancement and $s$-process elements in HE 0024-2523 are consistent with previous episodes of mass transfer. The observed upper limit of the lithium abundance $[\log \epsilon(\mathrm{Li})=+1.4$; see $\S$ 4.3.7] is quite in line with the observed upper limits of the Li-depleted stars discussed by Ryan et al. (2001; see their Fig. 2). The estimated $v \sin i$ of HE 0024-2523 is at the high end (though still consistent with) the range of the Ryan et al. (2002) stars. The orbital eccentricities of the Ryan et al. (2002) Li-depleted stars are low as well, $e \leq 0.3$ (one, $\mathrm{BD}+51^{\circ} 1817$, is very close to circular), as are the eccentricities of the field blue stragglers of Carney et al. (2001). In the case of the latter, $\langle e\rangle=0.11$. It is worth noting that several of the blue stragglers in the Carney et al. (2001) sample exhibit detectable axial rotation, on the order of $9-10 \mathrm{~km} \mathrm{~s}^{-1}$, similar to what we observe in HE 0024-2523. As has been emphasized by Ryan et al. (2002), such high rotational velocities would not be naively expected for (presumably) quite ancient stars of the halo population and must have resulted from some latestage spin-up, quite likely at the time of mass transfer.

The one observable that clearly distinguishes $\mathrm{HE}$ 0024-2523 from the stars studied by Ryan et al. (2002) is its extremely short orbital period, 3.4 days, as compared with the typical orbital period of 200-700 days in the latter sample. However, there are three field blue stragglers in the Carney et al. sample, CS 22170-028, CS 22873-139, and CS 22890-069, that possess orbital periods of from 1 to 20 days, similar to HE 0024-2523 (the rest of the Carney et. al. sample exhibit orbital periods that are substantially longer). The axial rotations of these three stars have not been measured. The case of CS 22873-139 is particularly interesting, as this star was shown to be a double-line spectroscopic binary by Preston (1994), who argued that at least the primary of this system might be identified with the "blue metal-poor main-sequence" (BMP) population of halo stars noted by Preston, Beers, \& Shectman (1994). These BMP stars are the very same population Preston \& Sneden (2000) showed to be dominated by binaries, presumably many of which either are or will become field blue stragglers. In a detailed high-resolution study by Spite et al. (2000), this star was shown to exhibit an unusual pattern of elemental abundances, with strikingly low $[\mathrm{Sr} / \mathrm{Fe}](<-1.1),[\mathrm{Mg} / \mathrm{Fe}]$ $(-0.04),[\mathrm{Ca} / \mathrm{Fe}](+0.16)$, and $[\mathrm{Al} / \mathrm{Fe}](-0.80)$, both compared with most other halo stars and with HE 0024-2523. There is no evidence of carbon enhancement in this star. The Li doublet at $6707 \AA$ was not detected, though Spite et al. argued that the abundance of lithium might still be consistent with the primordial value, based on the quality of their available data. Clearly, the abundance variations among the populations of stars that might be associated with the blue straggler phenomenon has yet to be fully explored.

\section{CONCLUSIONS}

The peculiarity of HE 0024-2523 and the comparison of its measured abundances and orbital parameters with other metal-poor stars in the literature suggest a correlation between binarity and observed $s$-process element patterns. This hypothesis is based on a very limited number of objects, and a larger sample of well-studied CEMP stars is required to improve our knowledge of the different evolutionary states, formation scenarios, and nucleosynthesis histories of these objects. To this end, we have already obtained spectra with HIRES of four more examples of apparent CEMP stars selected in the same way as described in Paper I of this series. We are aware of a number of other groups that are pursuing related observational campaigns, so the database of available information is expected to expand in the near future.

S. L., R. G, and E. C. acknowledge partial support from the MURST COFIN 2000. J. G. C and S. R. are grateful for partial support from the Fullham Award of the Dudley Observatory, and from grants AST 98-19614 and AST 0205951 awarded by the National Science Foundation. N. C. acknowledges financial support through a Marie Curie Fellowship of the European Community program Improving Human Research Potential and the Socio-Economic Knowledge under contract HPMF-CT-2001-01437, and from Deutsche Forschungsgemeinschaft under grant Re 353/44-1. T. C. B. acknowledges partial support for this work from grants AST 00-98508 and AST 00-98549 awarded by the National Science Foundation.

The authors are grateful to R. E. M. Griffin for supplying the ORBITSOLVER code. They also thank P. Bonifacio for performing part of the observations, and R. Gallino, F. D'Antona, P. Marigo and O. Straniero for useful discussions. 


\section{REFERENCES}

Anders, E., \& Grevesse, N. 1989, Geochim. Cosmochim. Acta, 53, 197

Aoki, W., et al. 2002a, PASJ, 54, 427

Aoki, W., Ando, H., Ryan, S. G., Beers, T. C., Ando, H., \& Tsangarides, S. 2003, ApJ, in press

Aoki, W., Norris, J. E., Ryan, S. G., Beers, T. C., \& Ando, H. 2000, ApJ, 536, L97

.2002b, ApJ, 567, 1166

Aoki, W., et al. 2001, ApJ, 561, 346

Bard, A.,'\& Kock, M. 1994, A\&A, 282, 1014

Baumüller, D., \& Gehren, T. 1997, A\&A, 325, 1088

Beers, T. C. 1999, in ASP Conf. Ser. 165, The Third Stromlo Symposium: The Galactic Halo, ed. B. Gibson, T. Axelrod, \& M. Putman (San Francisco: ASP), 202

Beers, T. C., Preston, G. W., \& Shectman, S. A. 1992, AJ, 103, 1987

Behr, B. 1999, Ph.D. thesis, California Inst. Tech.

Biémont, E., Garnir, H. P., Palmeri, P., Li, Z. S., \& Svanberg, S. 2000, MNRAS, 312,116

Biémont, E., Grevesse, N., Hannaford, P., \& Lowe, R. M. 1981, ApJ, 248, 867

Biémont, E., Hibbert, A., Godefroid, M., Vaeck, N., \& Fawcett, B. C. 1991, ApJ, 375, 818

Bizzarri, A., Huber, M. C. E., Noels, A., Grevesse, N., Bergeson, S. D., Tsekeris, P., \& Lawler, J. E. 1993, A\&A, 273, 707

Blackwell, D. E., Ibbetson, P. A., Petford, A. D., \& Shallis, M. J. 1979a, MNRAS, 186, 633

Blackwell, D. E., Menon, S. L. R., \& Petford, A. D. 1984, MNRAS, 207, 533

Blackwell, D. E., Menon, S. L. R., Petford, A. D., \& Shallis, M. J. 1982a, MNRAS, 201, 611

Blackwell, D. E., Petford, A. D., \& Shallis, M. J. 1979b, MNRAS, 186, 657

Blackwell, D. E., Petford, A. D., Shallis, M. J., \& Leggett, S. 1982b, MNRAS, 199, 21

Blackwell, D. E., Petford, A. D., Shallis, M. J., \& Simmons, G. J. 1980, MNRAS, 191, 445 1982c, MNRAS, 199, 43

Bonifacio, P., Molaro, P., Beers, T. \& Vladilo, G. 1998, A\&A, 332, 672

Booth, A. J., Shallis, M. J., \& Wells, M. 1983, MNRAS, 205, 191

Boothroyd, A. I., \& Sackmann, I.-J. 1988a, ApJ, 328, 632

. 1988b, ApJ, 328, 641

$1988 \mathrm{c}, \mathrm{ApJ}, 328,653$

. 1988d, ApJ, 328, 67

Brzozowski, J., Bunker, P., Elander, N., \& Erman, P. 1976, ApJ, 207, 414

Busso, M., Gallino, R., Lambert, D. L., Travaglio, C., \& Smith, V. V. 2001, ApJ, 557, 802

Carney, B. W., Latham, D. W., Laird, J. B., Grant, C. B., \& Morse, J. A. 2001, AJ, 122, 3419

Carretta, E., Gratton, R., Cohen, J. G., Beers, T. C., \& Christlieb, N. 2002, AJ, 124, 481 (Paper II)

Cayrel, R., et al. 2001, Nature, 409, 691

Christlieb, N., Green, P. J., Wisotzki, L., \& Reimers, D. 2001a, A\&A, 375, 366

Christlieb, N., Wisotzki, L., Reimers, D., Homeier, D., Koester, D., \& Heber, U. 2001b, A\&A, 366, 898

Cohen, J. G., Christlieb, N., Beers, T. C., Gratton, R. G., \& Carretta, E. 2002, AJ, 124, 470 (Paper I)

Dekker, H., D'Odorico, S., Kaufer, A., Delabre, B., \& Kotzlowski, H. 2000, Proc. SPIE, 4008, 534

Engleman, R., \& Rouse, P. E. 1975, J. Quant. Spectrosc. Radiat. Transfer, 15,831

Goriely, S., \& Siess, L. 2001, A\&A, 378, L25

Gratton, R. G., Carretta, E., Eriksson, E., \& Gustafsson, B. 1999, A\&A, 350,955

Griffin, R. F., \& Cornell, A. P. 1998, MNRAS, 293, 151

Hannaford, P., Lowe, R. M., Grevesse, N., Biemont, E., \& Whaling, W. 1982, ApJ, 261, 736

Hauge, Ø., \& Sørli, H. 1973, Sol. Phys., 30, 301

Herwig, F., Bloecker, T., Schoenberner, D., \& El Eid, M. 1997, A\&A, 324, L81
Hill, V., Barbuy, B., Spite, M., Spite, F., Plez, R. C. B., Beers, T., Nordström, B., \& Nissen, P. 2000, A\&A, 353, 557

Hill, V., et al. 2002, A\&A, 387, 560

Holweger, H., \& Müller, E. A. 1974, Sol. Phys., 39, 19

Iben, I., \& Renzini, A. 1983, ARA\&A, 21, 271

Käppeler, F., Gallino, R., Busso, M., Picchio, G., \& Raiteri, C. M. 1990a, ApJ, 354, 630

Käppeler, F., Zhao, W. R., Beer, H., \& Ratzel, U. 1990b, ApJ, 355, 348

Kurucz, R. L. 1995a, CD-ROM 18, SYNTHE Spectrum Synthesis Programs and Line Data (Cambridge: Smithsonian Astrophys. Obs.)

. 1995b, CD-ROM 23, Atomic Line Data (Cambridge: Smithsonian Astrophys. Obs.)

Kurucz, R. L., Furenlid, I., \& Brault, J. 1984, National Solar Observatory Atlas (Sunspot: Natl. Sol. Obs.)

Lambert, D. L. 1978, MNRAS, 182, 249

Lawler, J. E., Bonvallet, G., \& Sneden, C. 2001a, ApJ, 556, 452

Lawler, J. E., Wickliffe, M. E., den Hartog, E. A., \& Sneden, C. 2001b, ApJ, 563, 1075

Lejeune, T., Cuisinier, F., \& Buser, R. 1998, A\&AS, 130, 65

Lucatello, S., \& Gratton, R. G. 2002, A\&A, submitted

Magain, P. 1985, A\&A, 146, 95

Marigo, P. 1998, A\&A, 340, 463

Marigo, P., Girardi, L., \& Bressan, A. 1999, A\&A, 344, 123

McClure, R. D., \& Woodsworth, A. W. 1990, ApJ, 352, 709

McWilliam, A., Preston, G., Sneden, C., \& Searle, L. 1995, AJ, 109, 2757

Moore, C. E., Minnaert, M. G. J., \& Houtgast, J. 1966, The Solar Spectrum $2935 \AA$ to $8770 \AA$ (NBS Monogr. 61) (Washington: GPO)

Norris, J. E., Ryan, S. G., \& Beers, T. C. 1997a, ApJ, 488, 350

. 1997b, ApJ, 489, L169

Norris, J., Ryan, S., Beers, T., Aoki, W., \& Ando, H. 2002, ApJ, 569, L107

O’Brian, T. R., \& Lawler, J. E. 1991, Phys. Rev. A, 44, 7134

O'Brian, T. R., Wickliffe, M. E., Lawler, J. E., Whaling, J. W., \& Brault, W. 1991, J. Opt. Soc. Am. B, 8, 1185

Oke, J. G., \& Gunn, J. E. 1982, PASP, 94, 586

Preston, G. W. 1994, AJ, 108, 2267

Preston, G. W., Beers, T. C., \& Shectman, S. A. 1994, AJ, 108, 538

Preston, G. W., \& Sneden, C. 2000, AJ, 120, 1014 2001, AJ, 122, 1545

Ryan, S. G., Beers, T. C., Kajino, T., \& Rosolankova, K. 2001, ApJ, 547, 231

Ryan, S. G., Gregory, S. G., Kolb, U., Beers, T. C., \& Kajino, T. 2002, ApJ, 571, 501

Sandquist, E. L., Taam, R. E., Chen, X., Bodenheimer, P., \& Burkert, A. 1998, ApJ, 500, 909

Shortridge K. 1993, in Astronomical Data Analysis Software and Systems II, ASP Conf. Ser. 52, ed. R. J. Hannisch, R. J. V. Brissenden, \& J. Barnes (San Francisco: ASP), 219

Siess, L., Livio, M., \& Lattanzio, J. 2002, ApJ, 570, 329

Smith, G., \& Raggett, D. S. J. 1981, J. Phys. B, 14, 4015

Sneden, C., Cowan, J. J., Ivans, I. I., Fuller, G. M., Burles, S., Beers, T. C., \& Lawler, J. E. 2000, ApJ, 533, L139

Sneden, C., McWilliam, A., Preston, G. W., Cowan, J. J., Burris, D. L., \& Armosky, B. J. 1996, ApJ, 467, 819

Sneden, C., Preston, G., McWilliam, A., \& Searle, L. 1994, ApJ, 431, L27

Spite, M., Depagne, E., Nordström, B., Hill, V., Cayrel, R., Spite, F., \& Beers, T. C. 2000, A\&A, 360, 1077

Steffen, M. 1985, A\&AS, 59, 403

Thévenin, F., \& Idiart, T. P. 1999, ApJ, 521, 753

Van Eck, S., Goriely, S., Jorissen, A., \& Plez, B. 2001, Nature, 412, 793

Ventura, P., D’Antona, F., \& Mazzitelli, I. 2002, A\&A, 393, 215

Vogt, S. S., et al. 1994, Proc. SPIE, 2198, 362

Wiese, W., \& Martin, G. A. 1980, Wavelength and Transitions: Part II (NSRDS-NSB 68) (Washington: NBS)

Wisotzki, L., Christlieb, N., Bade, N., Beckmann, V., Köhler, T., Vanelle, C., \& Reimers, D. 2000, A\&A, 358, 77

Yi, S., Demarque, P., Kim, Y., Lee, Y., Ree, C. H., Lejeune, T., \& Barnes, S. 2001, ApJS, 136, 417 